\title{
ASSESSING THE IMPACT OF COVID-19 ON GLOBAL FOSSIL FUEL CONSUMPTION AND CO2 EMISSIONS
}

\author{
L. Vanessa Smith \\ Nori Tarui \\ Takashi Yamagatax
}

July 2020

The Institute of Social and Economic Research

Osaka University

6-1 Mihogaoka, Ibaraki, Osaka 567-0047, Japan 


\title{
Assessing the impact of COVID-19 on global fossil fuel consumption and $\mathrm{CO}_{2}$ emissions*
}

\author{
L. Vanessa Smith ${ }^{\dagger}$ \\ DERS, University of York, UK \\ Nori Tarui ${ }^{\ddagger}$ \\ Department of Economics, University of Hawaii \\ Takashi Yamagata ${ }^{\S}$ \\ DERS, University of York \& ISER, Osaka University
}

25 June 2020

\begin{abstract}
The shock to the global economy from COVID-19 is predicted to be faster and more severe than the 2008 global financial crisis and even the Great Depression. We assess its impact on global fossil fuel consumption and $\mathrm{CO}_{2}$ emissions over a two-year horizon. For this purpose we employ a global vector autoregressive (GVAR) model, which captures complex spatial-temporal interdependencies across countries due to the spread of the virus and associated international propagation of economic impact. The model makes use of a unique quarterly data set of coal, natural gas, and oil consumption, output and exchange rates, including global fossil fuel prices for 32 major $\mathrm{CO}_{2}$ emitting countries. We produce forecasts of coal, natural gas and oil consumption, conditional on GDP growth scenarios based on alternative IMF World Economic Outlook forecasts that were made before and after the outbreak. We also simulate the effect of a relative price change in fossil fuels, due to carbon pricing in all countries in the sample, on consumption and output. Our results show that fossil fuel consumption and $\mathrm{CO}_{2}$ emissions are expected to return to their pre-crisis levels, and even exceed them, within the two-year horizon despite the large reductions in the first quarter following the outbreak. Our forecasts anticipate more robust growth for emerging than for advanced economies. Recovery to the pre-crisis levels is expected even if another wave of pandemic occurs within a year. The results from our counterfactual carbon pricing scenario show that an increase in coal prices is expected to have a smaller impact on GDP than on fossil fuel consumption. Thus, the COVID-19 pandemic would not provide countries with a strong reason to delay climate change mitigation efforts.
\end{abstract}

JEL Classifications: C33, O50,P18,Q41,Q43,Q47

Keywords: COVID-19, $\mathrm{CO}_{2}$ emissions, fuel consumption, Global VAR (GVAR), conditional forecasts

\footnotetext{
${ }^{*}$ We are grateful to Julia Touza-Montero, Joao Madeira and Ron Smith for helpful discussions. We thank Sara Elsayed, Rui Lin and Ryo Mikami for excellent research assistance.

†vanessa.smith@york.ac.uk

${ }^{\ddagger}$ Corresponding author. Address: 2424 Maile Way, 518 Saunders Hall, Honolulu, HI 96822 USA. E-mail: nori@hawaii.edu

§akashi.yamagata@york.ac.uk. This authour gratefully acknowledges support from JSPS KAKENHI (18K01545, 15H05728).
} 


\section{Introduction}

After the first case of pneumonia of unknown cause in Wuhan, China, was reported at the end of December 2019, the spread of novel coronavirus (COVID-19) has been causing a worldwide public health emergency. Social isolation and lockdown, which have been enforced to slow down the virus spread, led to a virtual halt of major economic activities. The World Economic Outlook, published in April 2020 by the International Monetary Fund (IMF, 2020b), predicts that the global economy will shrink by 3 per cent in 2020. This forecast was further revised downwards to a 4.9 per cent shrink in June 2020 (IMF, 2020c). This means that the shock to the global economy from COVID-19 could be more severe than the 2008 global financial crisis and even the Great Depression.

According to latest research, the sharp drop in economic activity due to the enforced confinement has dramatically reduced energy use, and hence carbon dioxide $\left(\mathrm{CO}_{2}\right)$ emissions. Le Quere et al. (2020) estimated daily changes in global $\mathrm{CO}_{2}$ emissions taking account of the levels of the confinement policy. Based on the emissions data for six economic sectors across 69 countries, their results indicated a $17 \%$ decline in daily global $\mathrm{CO}_{2}$ emissions by early April 2020 relative to the mean level in 2019. Liu et al. (2020) report a decrease of $7.8 \%$ in global $\mathrm{CO}_{2}$ emissions due to fossil fuel use during the first quarter of 2020 relative to the first quarter of 2019 .

Despite growing evidence of the instantaneous impact on energy consumption and $\mathrm{CO}_{2}$ emissions, longer-term effects have not been well understood. Studying such effects is important because related evidence will provide policy makers with essential information to prepare post-COVID-19 economic recovery packages. These packages should be designed to achieve strong economic growth as well as the international emission targets agreed in COP21 in Paris 11 If significant $\mathrm{CO}_{2}$ emissions reduction continues beyond a quarter or two, then one could further investigate which sectors of the economy experience more sustained emissions reduction. In this case, sector-level investigations of relative declines in emissions and economic activities may provide useful insights for cost-effective $\mathrm{CO}_{2}$ emissions in the future. If the emissions reduction over a year or two is limited, however, then continued efforts for further emissions mitigation become more urgent. It is then of interest to investigate how carbon pricing of global scale affects the countries' economic performance and emissions.

In this work we assess the impact of the global economic shock from COVID-19 on global fossil fuel consumption and $\mathrm{CO}_{2}$ emissions over a two-year horizon. For this purpose, we employ the global vector autoregressive (GVAR) model of Pesaran et al. (2004), which is further developed in Dées et al. (2007). GVAR is a large multi-country, spatial-temporal model that allows for a high degree of interdependence and dynamics. Notably, it can control for unobserved global or foreign shocks that affect each country. This approach is critical for assessing the impact of COVID-19 because of the global scale of its spread and the associated economic effects. The spread of the virus that first hit China induced significant economic disruptions in Asia, Europe, North America and beyond. While the spread of the virus itself reduced domestic economic activities in each country, it also disrupted the global supply chain (Ivanov, 2020), which in turn amplified the negative economic effects across countries. GVAR takes into account such cross-country dependencies and dynamic macroeconomic effects. For reliable estimation of such a large dimensional model, we apply GVAR to a sufficient number of time-series observations across countries that cover a major part of the world economy.

A related strand of literature investigates the relationship between energy consumption and growth; see Kahouli (2019) for an extensive survey. Many of these studies employ a single country analysis, adopting VAR models and vector error correction (VEC) models (e.g. Bloch, 2012; Akpan and Akpan, 2012; Bozkurt and Akan, 2014), due to the relatively small number of observations (annual data since 1960). Recently, studies using panel data analysis have emerged in the literature. Omri and Kahouli (2014) and Saidi and Hammami (2015), among others, use cross-country panel data with a relatively large number of countries (around 60) for 22-23 years of observations. They estimate short dynamic panel data models with generalised method of moments (GMM). Cores and Sanders (2013) and Antonakakis et al. (2017) employ panel VAR models whilst Shahbaz et al. (2013) employ the autoregressive distributed lag (ARDL) bounds testing approach using longer panel data with around 40 observations. These panel data studies permit country heterogeneity only in a limited way without controlling for global common shocks or spatial dependence despite both of these featuring prominently in the recent panel data econometrics literature. Notably, our econometric approach can address all

\footnotetext{
${ }^{1}$ See Allan et al. (2020) for a proposition of such recovery packages.
} 
of these issues. Thus it is suitable for studying the intermediate-term economic impact of COVID-19, which must take into account the different transmission channels of the economic effects of the virus across countries, over time.

In this paper, the model makes use of a unique quarterly data set of coal, natural gas and oil consumption, output and exchange rates, including global fossil fuel prices, for 32 countries spanning the period 1984Q1-2014Q4 ${ }^{2}$ To the best of our knowledge, no such compiled quarterly disaggregated consumption data set is publicly available for the period of our interest, apart from an annual data set provided by British Petroleum (BP) and the World Bank, which is typically used in the literature to date. According to the $\mathrm{BP}$ data for the year 2018, $81 \%$ of the global $\mathrm{CO}_{2}$ emissions due to fossil fuel combustion was released by the 32 countries in our sample. To predict $\mathrm{CO}_{2}$ emissions, it is important to decompose the fossil fuel sources into coal, natural gas and oil because of the different $\mathrm{CO}_{2}$ intensity among fuel sources. Furthermore, among the fossil fuels, the share of coal consumption within emerging economies is much higher than in advanced economies. Our forecasts indicate how $\mathrm{CO}_{2}$ emissions evolve in the emerging and advanced economies as the fuel mix changes across countries within these groups.

In order to assess the impact of the global economic shock from the COVID-19 spread, we produce conditional forecasts of coal, natural gas and oil consumption, for the advanced and the emerging economies, separately, conditional on GDP forecast trajectories (scenarios) over eight quarters. These trajectories are based on the IMF forecasts for 2020Q1-2021Q4 published in IMF (2020b), the World Economic Outlook, April 2020. IMF (2020b) publishes the quarterly forecast figures, while the IMF (2020c) update does not. We compare the results of three scenarios to identify the effects of: $(0)$ no outbreak; (1) one virus spread in 2020Q1-Q2; (2) two waves of virus spread in 2020Q1-Q2 and 2021Q1-Q2. We assess the effects on the above two country groups individually as well as on the world as a whole.

Another main objective of this paper is to investigate the effect of changes in the relative fossil fuel prices on output and consumption. Carbon pricing policies impose costs to the combustion of fossil fuel. For example, as coal is more carbon intensive than natural gas, an introduction of, or tightening of, carbon pricing policy should increase the relative (end-user) price of coal. The effect of such (end-user) relative price changes on output is critical when assessing the trade-off between the reduction of emissions and the negative effect on the economy, which can be an important issue during this time of depression.

The above counterfactuals will allow us to assess the following for each country group (e.g. advanced and emerging): (i) relative emission resilience to the negative economic shock; (ii) relative sensitivity of emissions to carbon pricing; and (iii) relative robustness of output to carbon pricing. Related policy implications could be useful for the country- or regional-level long-term emissions targets beyond their intended nationally determined contributions under the Paris Agreement.

We find that fossil fuel consumption and $\mathrm{CO}_{2}$ emissions are expected to return to their pre-crisis levels, and even exceed them, within the two-year horizon despite large reductions in the first quarter following the outbreak. Our forecasts anticipate more robust recovery and growth for the emerging economies than for the advanced. Recovery to pre-crisis levels is expected even if another wave of pandemic takes place within a year. We argue that this result may be due to (i) the IMF forecasts predicting GDP recovery for the advanced economies in the two-year period, with faster recovery for the emerging economies, and (ii) limited responsiveness of fossil fuel consumption to income changes, as indicated in existing empirical studies.

With regard to fuel prices, they display a sharp decline over the first quarters before exhibiting recovery. Importantly, different fuels have different trajectories of price recovery. The coal and oil prices first increase then drop sharply before recovering in the second year. The fuel prices are expected to return to their pre-crisis level within the two-year period, but the price recovery may be slower if a second pandemic wave occurs within a year. Turning to the effect of carbon pricing, our counterfactual analysis suggests that (i) a permanent rise in the price of coal relative to natural gas and oil will reduce fossil fuel consumption in advanced and emerging economies by a similar magnitude; (ii) the negative effects on GDP are smaller than those on coal consumption; and (iii) the magnitude of the negative GDP effect on the emerging economies is only around half of what the advanced economies experience.

\footnotetext{
${ }^{2}$ The end date of $2014 \mathrm{Q} 4$ is due to data not being available primarily for natural gas for a number of countries in our sample, and also to the large time delay in providing data extensions at the primary source level, mainly for this variable.
} 
These results suggest that, while emerging economies appear to be more resilient to carbon pricing policy, such policy is as effective in reducing emissions for these economies as it is for the advanced ones.

Our research is not the first to employ the GVAR model in the area of energy economics. Cashin et al. (2014) apply a GVAR model for 38 countries/regions over the period 1979Q2-2011Q2 to show that the economic consequences of a supply-driven oil-price shock are very different from those of an oildemand shock driven by global economic activity. Mohaddes and Pesaran (2016) develop a GVAR-oil model that integrates a model for the global oil market within that of the global economy to identify country-specific oil-supply shocks. Mohaddes and Pesaran (2017), using a global VAR model for 27 countries/regions over the period 1979Q2 and 2013Q1, find that a fall in oil prices lowers interest rates and inflation in most countries and increases global real equity prices. Mohaddes and Raissi (2019) use a similar GVAR model to Cashin et al. (2014) to investigate the global macroeconomic consequences of falling oil prices due to the oil revolution in the USA. We are not aware of any GVAR analysis that focuses on energy consumption of fossil fuels (at the country level) and related CO2 emissions.

The paper is organised as follows. Section 2 describes the data. Section 3 discusses the econometric model and estimation method. Section 4 explains the implementation of the conditional forecasting, conditional on the GDP scenarios after the COVID-19 spread. Section 5 discusses the experiment associated with a higher relative coal price. Section 6 concludes. Details of the data sources, the GVAR model, and additional empirical results can be found in Appendices A-C.

\section{Data}

In order to assess the impact of the global economic shock due to the COVID-19 spread on world $\mathrm{CO}_{2}$ emissions, we consider a multi-country model that captures complex spatial-temporal interdependencies of the global economy and energy consumption. We employ a unique quarterly data set of coal, natural gas and oil consumption, output and exchange rates, including global fossil fuel prices, for 32 countries spanning the period 1984Q1-2014Q4. The countries included in the analysis are listed in Table 1. According to the BP data for the year 2018, China, India and the US emitted $28.7 \%, 15.7 \%$ and $7.6 \%$ of $\mathrm{CO}_{2}$, totaling $51.9 \%$ of the $81 \%$ of world $\mathrm{CO}_{2}$ emissions released by these 32 countries as mentioned earlier. Because coal is cheaper relative to oil and natural gas, it is the main primary energy source for many emerging economies, including China and India. In view of this, we explicitly use coal, natural gas and oil consumption data for each of the countries.

Following the IMF, we partition the countries into two groups, the advanced economies and the emerging market and developing economies, as shown in Table 1. To be concise, we refer to the latter group simply as 'emerging economies'. Within the advanced economies, we also consider the subset $\mathrm{EU}^{+}$, which consists of ten EU countries plus Norway and Switzerland. We include these two countries due to their historical close relationship to the EU. This group has been severely hit by the pandemic and is subject to the most stringent carbon pricing policy.

We analyse spatial-temporal interactions between eight variables. Let coal ${ }_{i t}, g s_{i t}$ and oil $_{i t}$ represent the logarithms of per capita consumption of coal, natural gas and crude oil (in Mtoe) for country $i$ in quarter $t$. The logarithm of the associated global prices, pcoal $l_{t}, p_{\text {gas }}$ and poil $_{t}$, are based on the Australian coal price (US dollars per mt), European Natural Gas price (US dollars per mmbtu) and Brent crude oil (US dollars per bbl), respectively, which are all normalised to 100 in 2014Q4. The remaining two domestic variables are economic variables, $g d p_{i t}$ and $e p_{i t}$, which are the logarithms of the (real) gross domestic product and the real exchange rate in terms of US dollars for country $i$ in quarter $t$, respectively. Details of the construction and sources of the data are available in Appendix A.1. The consumption data were first tested for seasonality. Those series that exhibited significant seasonal effects were subsequently temperature adjusted and/or seasonally adjusted. In some cases where temperature adjustment induced spurious volatility, only seasonal adjustment was performed. Temperature adjustment is based on the use of heating and cooling degree days as well as their 30-year average between 1981 and 2010 (the 'climate normals,' see Elkhafif, 1996, and Won et al., 2016, for recent applications). The procedures for performing temperature and seasonal adjustment as well as for assessing seasonal effects can be found in Appendix A.2, together with a table summarising the required adjustments to the consumption series for each country. Energy prices as well as the economic variables (in the case of $e p$ the underlying Consumer Price Indices) were seasonally adjusted where 
Table 1: 32 countries and associated groups within the sample

\begin{tabular}{|l|l|l|}
\hline \multicolumn{2}{|c|}{ Advanced Economies } & Emerging Economies \\
\hline \multicolumn{1}{|c|}{ EU $^{+}$} & & \\
\hline Austria & Australia & Argentina \\
Belgium & Canada & Brazil \\
France & Japan & China \\
Finland & Korea & Chile \\
Germany & New Zealand & India \\
Italy & Singapore & Indonesia \\
Netherlands & United States & Malaysia \\
Norway & & Mexico \\
Spain & & Philippines \\
Sweden & & South Africa \\
Switzerland & & Saudi Arabia \\
United Kingdom & & Thailand \\
& & Turkey \\
\hline
\end{tabular}

required.

\section{Modelling Framework}

We employ the global vector autoregressive (GVAR) modelling framework of Pesaran et al. (2004), which is further developed in Dées et al. (2007). GVAR is based on a multi-country model that links country-specific models in a coherent manner using time series and panel techniques. The ultimate aim of the GVAR approach is to construct a VAR model formed of a global variable vector, which consists of the endogenous variables of all the countries. Define the $k_{i} \times 1$ vector of energy consumption and economic variables of country $i$ in quarter $t$ by $\mathbf{x}_{i t}=\left(\text { coal }_{i t}, g a s_{i t}, o i l_{i t} ; g d p_{i t}, e p_{i t}\right)^{\prime}$, as well as the $m_{d} \times 1$ vector of energy prices by $\mathbf{d}_{t}=\left(\text { pcoal }_{t}, \text { pgas } t, \text { poil }_{t}\right)^{\prime}$. Stacking $\mathbf{x}_{i t}$ for $i=0,1, \ldots, N$ for our 32 countries yields the $k \times 1$ global variable vector, $\mathbf{x}_{t}=\left(\mathbf{x}_{0 t}^{\prime}, \mathbf{x}_{1 t}^{\prime}, \ldots, \mathbf{x}_{N t}^{\prime}\right)^{\prime}$ with $k=\sum_{j=0}^{N} k_{i}$, and country 0 taken as the numeraire country, here the US. The $p^{t h}$-order GVAR model of our $155 \times 1^{3}$ global variable vector and energy prices at $t, \mathbf{y}_{t}=\left(\mathbf{x}_{t}^{\prime}, \mathbf{d}_{t}^{\prime}\right)^{\prime}$, is given by

$$
\mathbf{y}_{t}=\mathbf{c}_{0}+\mathbf{c}_{1} t+\mathbf{C}_{1} \mathbf{y}_{t-1}+\ldots+\mathbf{C}_{p} \mathbf{y}_{t-p}+\varepsilon_{t}, t=1, \ldots, T
$$

where contemporaneous correlations in the error term are permitted. The GVAR model of equation (1) is a large model that, despite its simple overall structure, allows for a rich set of dynamics including a high degree of interdependencies. It is not directly estimable due to the curse of dimensionality and possible existence of cointegrated variables. To avoid these problems, estimation and specification of the GVAR model involves two-steps. In the first step, a vector error correction model for the domestic variables, $\mathbf{x}_{i t}$, is estimated for each $i$, augmented with the global variables, $\mathbf{d}_{t}$, and the foreign variables of country $i, \mathbf{x}_{i t}^{*}$, which are specified below. Based on these parameter estimates as well as those of an estimated model for $\mathbf{d}_{t}$, in the second step the estimated version of equation (1) is obtained. The two-step estimation approach is explained in detail below.

\subsection{The country-specific VARX* models}

Consider the following $\operatorname{VARX} *\left(p_{i}, q_{i}\right)$ structure for the $i^{\text {th }}$ country-specific model

$$
\begin{aligned}
\mathbf{x}_{i t} & =\mathbf{a}_{i 0}+\mathbf{a}_{i 1} t+\boldsymbol{\Phi}_{i 1} \mathbf{x}_{i, t-1}+\ldots+\boldsymbol{\Phi}_{i p_{i}} \mathbf{x}_{i, t-p_{i}}+\boldsymbol{\Lambda}_{i 0} \mathbf{x}_{i t}^{*}+\Lambda_{i 1} \mathbf{x}_{i, t-1}^{*}+\ldots+\boldsymbol{\Lambda}_{i q_{i}} \mathbf{x}_{i, t-q_{i}}^{*}, \\
& +\mathbf{\Psi}_{i 0} \mathbf{d}_{t}+\mathbf{\Psi}_{i 1} \mathbf{d}_{t-1}+\ldots+\mathbf{\Psi}_{i q_{i}} \mathbf{d}_{t-q_{i}}+\mathbf{u}_{i t}
\end{aligned}
$$

\footnotetext{
${ }^{3}$ See Table B.1(ii) in Appendix B.1.
} 
for $i=0,1, \ldots, N$ where $\mathbf{x}_{i t}$ and $\mathbf{d}_{t}$ are the $k_{i} \times 1$ vector of domestic variables and the $m_{d} \times 1$ vector of common global variables, respectively, given above, and $\mathbf{x}_{i t}^{*}$ is a $k_{i}^{*} \times 1$ vector of foreign variables. The foreign variables are constructed as weighted averages across all domestic variables in the model such that $\mathbf{x}_{i t}^{*}=\sum_{j=0}^{N} w_{i j} \mathbf{x}_{j t}$, where $w_{i i}=0$ and $\sum_{j=0}^{N} w_{i j}=1$, and can be considered as proxies for unobserved common factors. These variables are expected to 'soak up' most of the cross-section correlation leaving only a modest degree in the estimated residuals. The weights, $w_{i j}$, are computed here based on the trade relationship (average of imports and exports) of the individual countries with their corresponding trading partners $4_{4}^{4}$ The common global variables in each country model are treated similar to the foreign 'star' variables, which includes sharing the same lag order, $q_{i}$.

As discussed in Pesaran et al. (2004), GVAR allows for interactions among different countries through three separate but interrelated channels:

1. Contemporaneous dependence of $\mathbf{x}_{i t}$ on $\mathbf{x}_{i t}^{*}$ and on its lagged values, where as mentioned earlier the star variables can be considered as proxies for common unobserved factors such as, for example, the diffusion of technological progress or global upheaval in the case of COVID-19.

2. Dependence of the country-specific variables $\mathbf{x}_{i t}$ on common global variables $\mathbf{d}_{t}$ and on its lagged values, which are the global fuel prices in our context.

3. Nonzero contemporaneous dependence of shocks in country $i$ on the shocks in country $j$, measured via the cross-country covariances, $\boldsymbol{\Sigma}_{i j}=\operatorname{cov}\left(\mathbf{u}_{i t}, \mathbf{u}_{j t}\right)$ for $i \neq j$. Such 'residual' interdependencies (after global unobserved factors have been taken into account) could be due, for example, to policy and trade spillover effects.

In the presence of possible $I(1)$ variables and cointegration the corresponding vector error correction form of equation (2), the $\mathrm{VECMX}^{*}$, assuming for expositional purposes that $p_{i}=q_{i}$, can be written as

$$
\Delta \mathbf{x}_{i t}=\mathbf{c}_{i 0}-\boldsymbol{\alpha}_{i} \boldsymbol{\beta}_{i}^{\prime}\left[\widetilde{\mathbf{z}}_{i, t-1}-\gamma_{i}(t-1)\right]+\boldsymbol{\Lambda}_{i 0} \Delta \mathbf{x}_{i t}^{*}+\mathbf{\Psi}_{i 0} \Delta \mathbf{d}_{t}+\sum_{j=1}^{p_{i}-1} \boldsymbol{\Gamma}_{z, i j} \Delta \widetilde{\mathbf{z}}_{i, t-j}+\mathbf{u}_{i t},
$$

where $\mathbf{z}_{i t}=\left(\mathbf{x}_{i t}^{\prime}, \mathbf{x}_{i t}^{* \prime}\right)^{\prime}, \widetilde{\mathbf{z}}_{i, t-1}=\left(\mathbf{z}_{i, t-1}^{\prime}, \mathbf{d}_{t-1}^{\prime}\right)^{\prime}, \boldsymbol{\alpha}_{i}$ is a $k_{i} \times r_{i}$ matrix of rank $r_{i}$ and $\boldsymbol{\beta}_{i}$ is a $\left(k_{i}+k_{i}^{*}+m_{d}\right) \times r_{i}$ matrix of rank $r_{i}$. By partitioning $\boldsymbol{\beta}_{i}$ as $\boldsymbol{\beta}_{i}=\left(\boldsymbol{\beta}_{i x}^{\prime}, \boldsymbol{\beta}_{i x *}^{\prime}, \boldsymbol{\beta}_{i d}^{\prime}\right)^{\prime}$ conformable to $\tilde{\mathbf{z}}_{i t}=\left(\mathbf{x}_{i t}^{\prime}, \mathbf{x}_{i t}^{* \prime}, \mathbf{d}_{t}^{\prime}\right)^{\prime}$, the $r_{i}$ error correction terms defined by the above equation can be written as

$$
\boldsymbol{\beta}_{i}^{\prime}\left(\widetilde{\mathbf{z}}_{i t}-\boldsymbol{\gamma}_{i} t\right)=\boldsymbol{\beta}_{i x}^{\prime} \mathbf{x}_{i t}+\boldsymbol{\beta}_{i x *}^{\prime} \mathbf{x}_{i t}^{*}+\boldsymbol{\beta}_{i d}^{\prime} \mathbf{d}_{t}-\left(\boldsymbol{\beta}_{i}^{\prime} \gamma_{i}\right) t
$$

which allows for the possibility of cointegration both within $\mathbf{x}_{i t}$ and between $\mathbf{x}_{i t}, \mathbf{x}_{i t}^{*}$ and $\mathbf{d}_{t}$, and consequently across $\mathbf{x}_{i t}$ and $\mathbf{x}_{j t}$ for $i \neq j$.

For the estimation of (2) the foreign and common global variables are treated as weakly exogenous with respect to the long run parameters $\boldsymbol{\alpha}_{i}$ and $\boldsymbol{\beta}_{i}$ of the model, an assumption testable by the data. This assumption implies that there are no feedback effects from the domestic variables to these variables in the long-run without precluding short-term interactions between the two. It is effectively this assumption that allows the GVAR model to decouple and for the countries to be estimated individually, conditional on the foreign and global variables. Further details on the variables included in the GVAR model are found in Appendix B.1.

The rank of the cointegrating space for each country model is computed using Johansen's (1991) trace statistic as set out in Pesaran et al. (2000) for models with weakly exogenous $I(1)$ regressors. This way, the number of cointegrating relations of each country, $r_{i}$, the speed of adjustment coefficients, $\boldsymbol{\alpha}_{i}$, and the cointegrating vectors $\boldsymbol{\beta}_{i}$ for each country model are obtained. Conditional on a given estimate of $\boldsymbol{\beta}_{i}$, the remaining parameters in equation (3) are consistently estimated by ordinary least squares (OLS). From the estimated VECMX* models we can then recover the estimated version of equation (2).

Unit root tests applied to our variables suggested that on the whole these are $I(1)$. The lag orders of the individual country VARX* models were selected using the Akaike information criterion (AIC) setting a maximum for $p_{i}$ and $q_{i}$ of 3 and 2 , respectively. The choice of these maximum values was based on the available number of observations and the desire to reduce serial correlation. The testable

\footnotetext{
${ }^{4}$ In principle any type of weights could be used including different weights for different variables, as well as weights that change over time, as long as they satisfy the 'smallness' condition given in Pesaran et al. (2004).
} 
assumption of weak exogeneity of the foreign and global variables was supported by the data. Weak exogeneity test results for all countries, along with the individual country lag orders, number of cointegrating relations and other related output can be found in Section S.2 of the supplementary material. The construction of the weight matrix is based on a three year average of the trade relationships between the countries over the years 2008-2010.

\subsection{Modelling the global prices}

While estimation of the individual country VECMX* models in the presence of the weakly exogenous regressors does not require separately specifying a model for the global prices, for the purpose of forecasting in what follows this is required.

The modelling procedure for the global prices proceeds in two steps. Since the global prices, $\mathbf{d}_{t}$, were found to be $I(1)$ in order to allow for the possibility of cointegration, in the first step the following error correction model that includes a restricted intercept is estimated

$$
\Delta \mathbf{d}_{t}=-\boldsymbol{\alpha}_{d} \boldsymbol{\beta}_{d}^{\prime}\left[\mathbf{d}_{t-1}-\boldsymbol{\mu}\right]+\sum_{j=1}^{p_{d}-1} \boldsymbol{\Gamma}_{d, j} \Delta \mathbf{d}_{t-j}+\boldsymbol{\eta}_{t},
$$

where $\boldsymbol{\alpha}_{d}$ and $\boldsymbol{\beta}_{d}$ are $m_{d} \times r_{d}$ vectors, and $r_{d}$ denotes the number of cointegrating relations. Using the trace statistic of the Johansen cointegration approach one cointegrating relationship was found among the global prices, with $p_{d}=3$ selected based on the AIC and no remaining serial correlation.

The cointegrating vector, $\boldsymbol{\beta}_{d}$, was estimated subject to one overidentifying restriction which was supported by the data. Let $\widehat{E C}_{d, t-1}=\hat{\boldsymbol{\beta}}_{d}^{\prime}\left[\mathbf{d}_{t-1}-\hat{\boldsymbol{\mu}}\right]$ be the estimated error correction term which is given by

$$
\widehat{E C}_{d, t-1}=0.267 \text { coal }_{t-1}+1.000 \text { gas }_{t-1}-1.000 \text { oil }_{t-1}-1.347 .
$$

In the second step the error correction specification in equation (4) is augmented with additional feedback effects computed as a weighted average of the domestic variable vector, $\mathbf{x}_{i t}$. Specifically,

$$
\Delta \mathbf{d}_{t}=\mathbf{c}+\boldsymbol{\delta} \widehat{E C}_{d, t-1}+\sum_{j=1}^{\widetilde{p}-1} \boldsymbol{\Theta}_{d, j} \Delta \mathbf{d}_{t-j}+\sum_{j=1}^{\widetilde{q}-1} \boldsymbol{\Theta}_{x, j} \Delta \widetilde{\mathbf{x}}_{t-j}+\boldsymbol{\eta}_{t}
$$

where $\widehat{E C}_{d, t-1}$ is taken as given (estimated in the first step), $\widetilde{\mathbf{x}}_{t}=\sum_{i=0}^{N} \widetilde{w}_{i} \mathbf{x}_{i t}$ is a $k \times 1$ vector of feedback effects, with the weights $\widetilde{w}_{i}$ such that $\sum_{i=0}^{N} \widetilde{w}_{i}=1$, which are computed based on PPP-GDP figures averaged over the years 2008-2010.

We further allow for separate lag orders, namely $\widetilde{p}_{\ell}$ and $\widetilde{q}_{\ell}$ with $\ell=1,2,3$, to be selected by the AIC for each of the individual price equations using a maximum lag order of 3 for both. Having estimated equation (6) the VAR form for $\mathbf{d}_{t}$ is given by

$$
\mathbf{d}_{t}=\boldsymbol{\mu}_{0}+\boldsymbol{\mu}_{1} t+\boldsymbol{\Phi}_{d, 1} \mathbf{d}_{t-1}+\ldots+\boldsymbol{\Phi}_{d, \widetilde{p}} \mathbf{d}_{t-\widetilde{p}}+\boldsymbol{\Lambda}_{x, 1} \widetilde{\mathbf{x}}_{t-1}+\ldots+\boldsymbol{\Lambda}_{x, \widetilde{q}} \widetilde{\mathbf{x}}_{t-\widetilde{q}}+\boldsymbol{\eta}_{t} .
$$

Combining the estimated versions of models (2) and (7), the estimated GVAR model of equation (1) in terms of $\mathbf{y}_{t}$ is obtained. Details of solving for the GVAR model based on equations (2) and (7) can be found in Appendix B.2.

\section{Conditional forecasts of fossil fuel consumption and carbon emis- sions}

In what follows, we produce forecasts for coal, natural gas and oil consumption conditional on three different eight-quarter horizon GDP growth rate paths (scenarios) for the advanced and emerging economies. These trajectories are based on the forecasts published in the IMF World Economic Outlook. We consider three GDP scenarios (Figure 1). Scenario 0 is the GDP forecast published by the IMF in January 2020, IMF (2020a), i.e., without the effect of the economic shock from the COVID-19 pandemic. Period zero corresponds to 2019Q4 and the value is normalised to 100. Scenario 1 is identical to the updated IMF forecast in April 2020 (IMF, 2020b, Figure 1.6, p.9), which clearly shows the effect of the economic shock from COVID-19's hit to Asia during January-February 2020 and to Europe and the US during March-April 2020. This trajectory asserts that the world economy returns to the long-run growth path in around 2020Q4, in the absence of a second outbreak. Scenario 

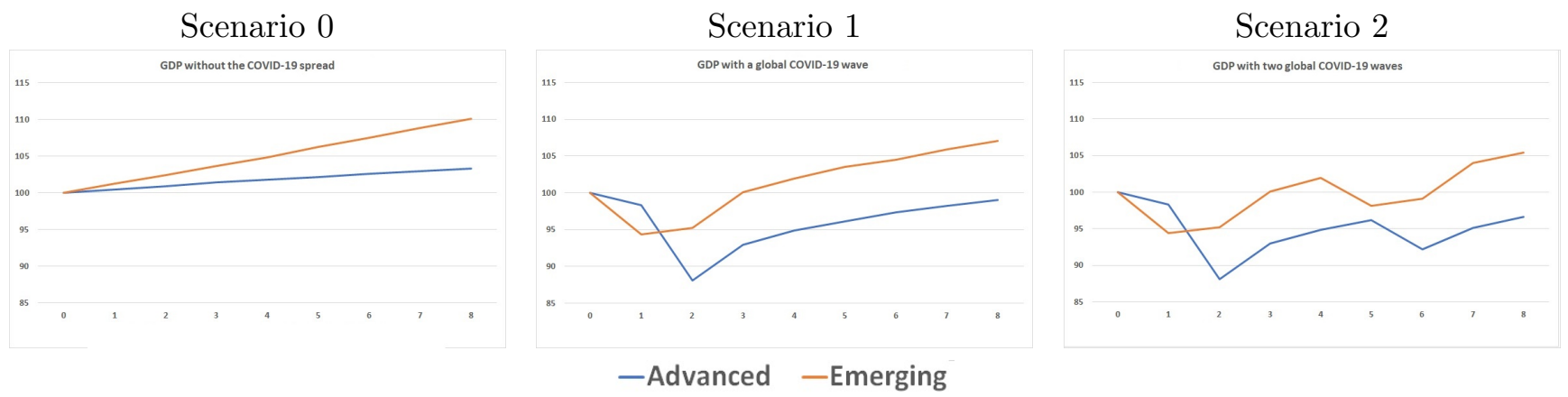

Figure 1: Three GDP scenarios with and without the global economic shock from COVID-19

2 assumes a second wave of pandemic around 2021Q1 and Q2 with an associated decline in GDP growth, which is slightly smaller in magnitude than that of the first wave.

In order to assess the effects of the global economic shock due to COVID-19, we implement conditional forecasting based on the growth rate paths of GDP indicated by the above scenarios. Technical details of this undertaking are explained in the next sub-section. In particular, we compute the eightquarter GDP trajectories corresponding to the above Scenarios 0-2 for each country of our model starting from the 2014Q4 GDP values, by applying the implied GDP changes of these scenarios, which are different for advanced and emerging economies.

A few remarks are warranted on this method of constructing the conditioning GDP scenarios. First, due to the availability of our data set ending at 2014Q4 and not 2019Q4, the estimated GDP trend and hypothesised GDP trend in the advanced and emerging groups may be slightly different 5 Second, even though the GDP growth rates would differ across individual countries, we assume a common GDP growth trend across countries within each of the two groups following the available published IMF forecasts. This assumption may be innocuous to the extent that our primary aim is to assess the impact of the (almost) simultaneous spread of COVID-19 to the advanced and emerging economies by comparing the conditional forecasts under Scenario 0 to those under Scenarios 1 and 2. We partially address this concern by further comparing the conditional forecasts with the unconditional forecasts from the GVAR model (1) in Appendix C.1. Finally, the IMF published revised GDP forecasts on 24 June 2020 (IMF, 2020c; Figure 1), in which the drop in 2020Q2 is larger for both the advanced and emerging groups compared to their April 2020 forecasts (IMF, 2020b). Unfortunately, unlike IMF (2020b), the revised quarterly forecast figures are not publicly available and we could not update our exercise. However, because the IMF's (2020c) revised GDP forecast falls between our Scenario 1 and Scenario 2 forecasts, it is likely that the forecasts of fossil fuel consumption and $\mathrm{CO}_{2}$ emissions under this revised Scenario 1 would be lower than those under our entertained (IMF, 2020b) Scenario 1 but higher than those under Scenario 2. Therefore, the general results in this paper would still hold with the revised scenario in IMF (2020c).

\subsection{Conditional forecasting: method}

Consider the estimated GVAR(3) model given by

$$
\mathbf{y}_{t}=\hat{\mathbf{c}}_{0}+\hat{\mathbf{c}}_{1} t+\hat{\mathbf{C}}_{1} \mathbf{y}_{t-1}+\hat{\mathbf{C}}_{2} \mathbf{y}_{t-2}+\hat{\mathbf{C}}_{3} \mathbf{y}_{t-3}+\hat{\boldsymbol{\varepsilon}}_{t}
$$

$t=1, \ldots, T$. Note that the estimation sample ends at 2014Q4. We produce conditional forecasts from model (8) for each quarter $T+h$, for $h=1,2, \ldots, 8$ conditional on the IMF forecasted GDP growth paths of 2020Q1-2021Q4 implied by each of the Scenarios 0-2.

The GDP growth paths are applied to the $g d p$ variable of all countries in model (8) starting from the last quarterly observation of our sample, $T$. This results in eight quarterly values for the $g d p$ variable for every country: namely, $g d p_{i, T+j}, j=1, \ldots, H(=8)$ (two different sets of values for the advanced and emerging economies). These can be written compactly as

$$
\mathbf{S y}_{T+j}=\mathbf{g}_{T+j}, j=1,2, \ldots, H,
$$

\footnotetext{
${ }^{5}$ As an alternative the GDP trend of $2014 \mathrm{Q} 4$ could be used.
} 
where $\mathbf{S}$ is a suitably defined $(N+1) \times(k+3)$ matrix with 1 in the position of $g d p_{i, T+j}$ for each country $i, 0$ elsewhere; $\mathbf{g}_{T+j}$ is a $(N+1) \times 1$ vector that contains the values for the $g d p$ variable corresponding to quarter $T+j$ for a given scenario; and $H$ denotes the conditioning horizon, which is equal to eight (the same as the forecast horizon). The forecast for every quarter, $T+h$, is then obtained by conditioning on all eight quarterly values for $g d p$ defined by (9) across all countries simultaneously.

The conditional point forecasts of $\mathbf{y}_{T+h}$ are given by

$$
\boldsymbol{\mu}_{h}^{*}=E\left(\mathbf{y}_{T+h} \mid \mathcal{I}_{T}, \mathbf{S y}_{T+j}=\mathbf{g}_{T+j}, j=1,2, \ldots, H\right), \text { for } h=1,2, \ldots, H,
$$

where $\mathcal{I}_{T}$ is the information set at time $T$. In deriving the expectations it is assumed that conditioning on the GDP growth paths does not affect the GVAR parameters, $\mathbf{C}_{i}, i=1,2,3$ and the covariance matrix, $\boldsymbol{\Sigma}_{\varepsilon}$, associated with $\boldsymbol{\varepsilon}_{t}$, which is also assumed to be jointly normally distributed.

We further define the unconditional point forecasts of $\mathbf{y}_{T+h}$ given by

$$
\boldsymbol{\mu}_{h}=E\left(\mathbf{y}_{T+h} \mid \mathcal{I}_{T}\right), \text { for } h=1,2, \ldots, H .
$$

While these forecasts condition on the information set $\mathcal{I}_{T}$, we define them as unconditional to distinguish them from the conditional forecasts in (10), which in addition condition on the $g d p$ values given by $\mathbf{S y}_{T+j}=\mathbf{g}_{T+j}, j=1,2, \ldots, H$.

We use the latter forecasts to construct the difference, $\boldsymbol{\delta}_{h}=\boldsymbol{\mu}_{h}^{*}-\boldsymbol{\mu}_{h}$. Obtaining the probability distribution of this difference then allows us to compute the probability, for example, that consumption is lower in view of Scenarios 1 and 2, and to check whether these probabilities are in line with the differences of the point forecasts obtained between Scenarios 1-0 and 2-0. It is the probabilities associated with $\boldsymbol{\delta}_{\boldsymbol{h}}$ that are given in Appendix C.1 referred to earlier. Further technical details related to the derivation of the conditional and unconditional forecasts, as well as the difference between the two, are available in Section $\mathbf{S . 3}$ of the supplementary material.

\subsection{Conditional forecasting: results}

In this subsection we report the conditional forecast results of the total amount of energy consumption and $\mathrm{CO}_{2}$ emissions for the different groups (see Table 1) under the different GDP scenarios.

\subsubsection{Energy consumption}

The conditional forecasts of the total amount of energy consumption are summarised in Figure2. The horizontal axis represents the quarters over the two-year forecast horizon and the vertical axis reports the forecasts of the amount of consumption. We first focus on the results for Scenario 0 (no COVID-19 effects). In the initial period (the observed 2014Q4 data point), the $\mathrm{EU}^{+}$and the advanced groups have a similar composition of energy consumption. In 2014Q4 the shares of coal, natural gas and oil for the advanced economies are $18.2 \%, 36.8 \%$ and $45 \%$, respectively. For the $\mathrm{EU}^{+}$countries, perhaps reflecting their stringent emission policy, the coal share is smaller $(13.7 \%)$ and the natural gas share is larger $(40.1 \%)$. In contrast, coal is the major energy source in the emerging group, which accounts for $57.2 \%$ of the total. Under Scenario 0, total fuel consumption in the emerging economies rises much faster than in the advanced over the eight-quarter horizon. This reflects the different average GDP growth rate of the two groups. The fuel mix also exhibits a different pattern of change for these two groups. For the advanced economies, the share of coal and oil decreases by $1.0 \%$ and $0.8 \%$, respectively, across the two-year horizon, which is compensated by a $1.8 \%$ rise in the share of natural gas. For the emerging group, the share of coal increases by $1.8 \%$ over the horizon.

We next turn to the results of Scenario 1 which assumes a one-time spread of COVID-19. The EU ${ }^{+}$ group appears to be hit much harder than the advanced group as a whole in the first quarter, displaying large negative drops in oil and natural gas consumption, simultaneously. Advanced economies as a whole follow a similar, but less pronounced pattern compared to $\mathrm{EU}^{+}$. This is followed by fast recovery of consumption in the subsequent quarter(s), and a further up and down movement in consumption. This implies that the observed effects of the COVID-10 outbreak in the first year may continue during the second forecast year even though patterns from this GDP scenario are stable at the end of the first year. The emerging economies exhibit a notable negative hit in the first quarter, but then start growing at a similar pace as under Scenario 0. Under Scenario 2, which assumes a resurgence of the 
coronarivus, the consumption patterns in $\mathrm{EU}^{+}$are similar over the first three quarters. The plunge in the fourth quarter is deeper and the recovery in the subsequent quarter is much weaker than in Scenario 1. A similar observation applies to the advanced economies. For emerging economies, the second wave of negative GDP shocks push down consumption in the fifth quarter, which drags down consumption growth.
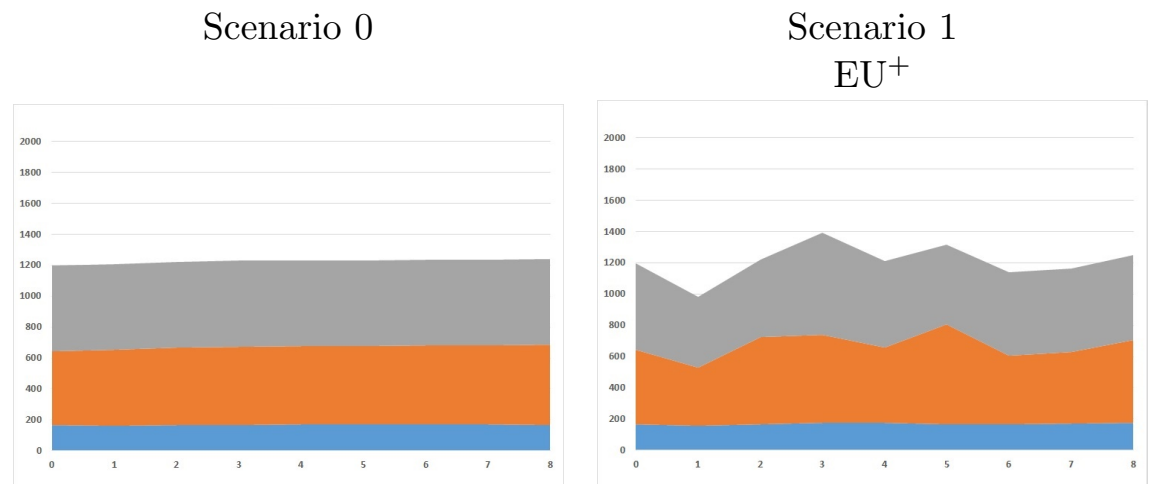

Scenario 2

Advanced
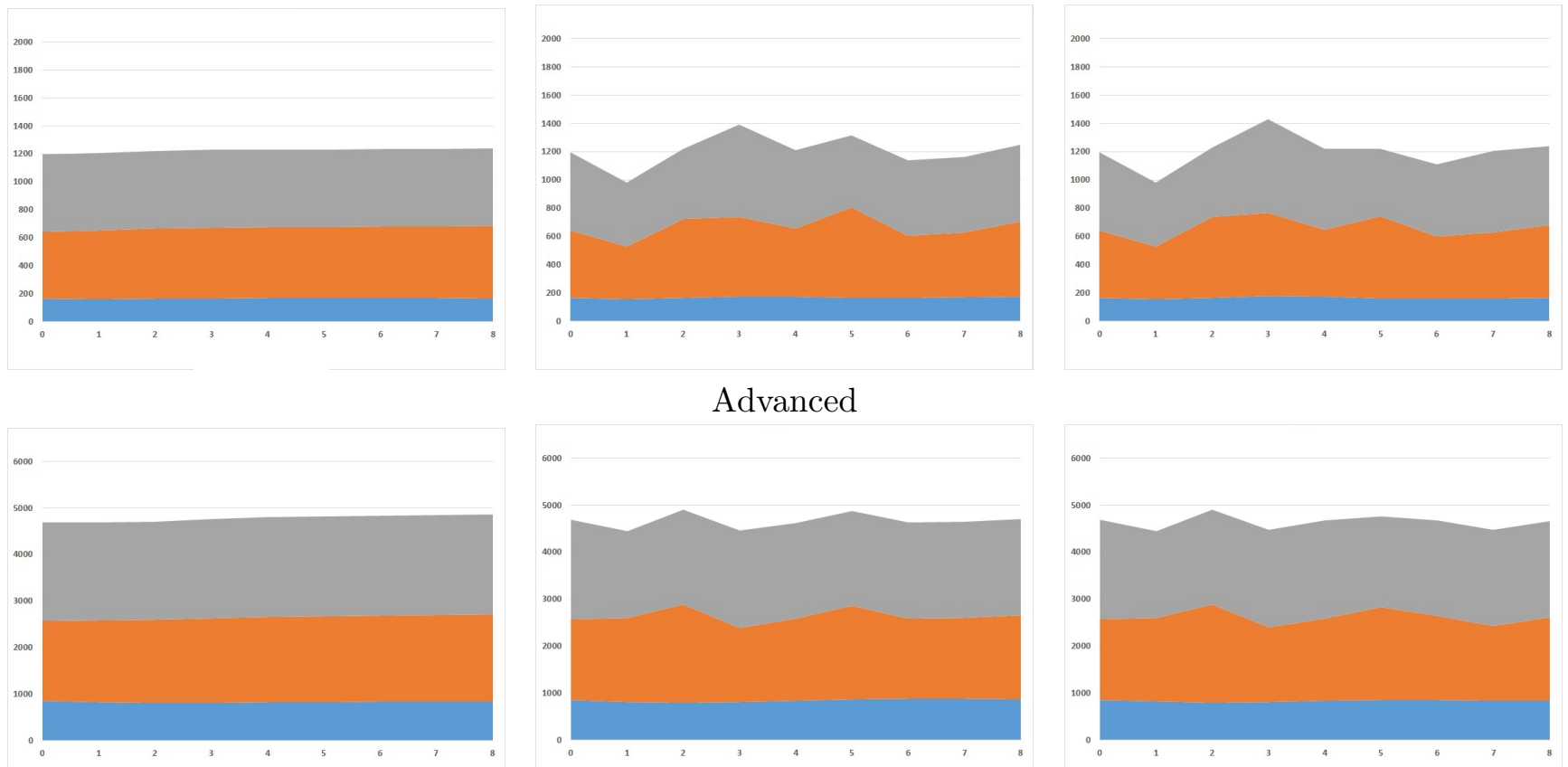

Emerging
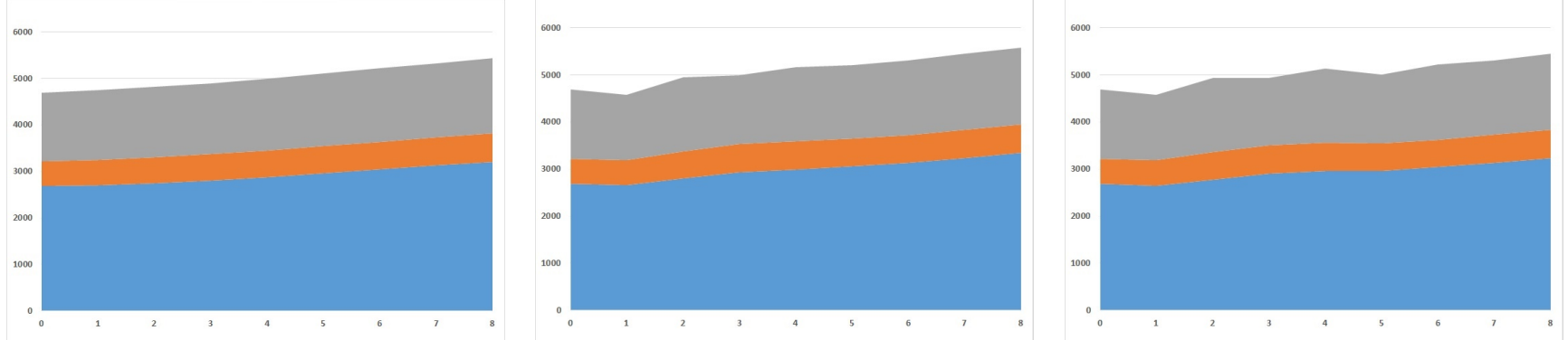

World
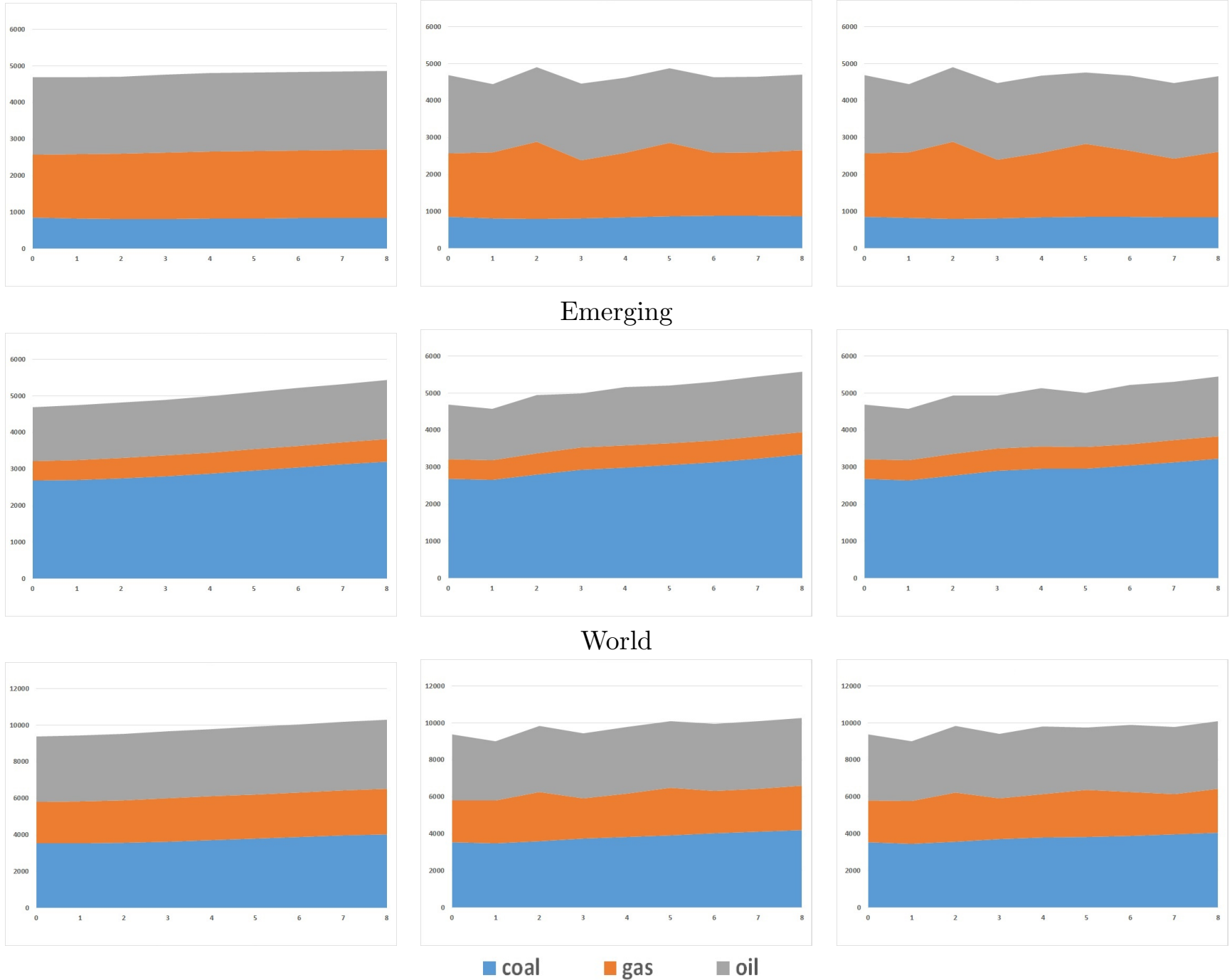

Figure 2: Forecasts of consumption of coal, natural gas, and oil by country group (annualised, in Mtoe) 


\subsection{2 $\quad \mathrm{CO}_{2}$ emissions}

Based on the forecasted energy consumption levels, we estimate the amount of emissions due to fossil fuel combustion. We use the simple emission factors which were used in the BP report. Specifically, a tonne of oil equivalent (toe) coal, natural gas and oil is converted to 3.96, 2.35 and 3.07 tonnes of $\mathrm{CO}_{2}{ }^{6}$

As expected, the emissions forecasts are qualitatively similar to the consumption forecasts as the former are a scaled version of the latter, weighted by the above emission factors. To save space, the emission forecasts are reported in Figure C.2 in Appendix C.2. Here we focus on investigating the effect of COVID-19's negative economic shock on emissions for the different country groups. We measure the effect by the change of each fuel sourced emissions implied by Scenario 1 or 2 (with a COVID-19 shock) over Scenario 0 (without the shock), which is reported in Figure 3 . The figures in the first row report the difference between the emissions in Scenario 1 and 0. For the advanced economies, the Scenario 1 GDP shock reduces emissions due to oil use across all eight quarters, whilst emissions due to coal and natural gas decrease and then increase continuously over the quarters. It is clear that oil is the largest contributor to the reduction of emissions. Table 2 reports the changes of the emissions for Scenarios 1 and 2 against Scenario 0. On average, over the eight quarters, the Scenario 1 GDP shock reduces emissions by $2.8 \%$ in advanced economies. On the other hand, in the emerging economies, apart from the drop in the first quarter, the emissions in the rest of the quarters are greater in Scenario 1 than in Scenario 0. The increase in emissions is mostly due to higher coal consumption. The average change in emissions for Scenario 1 over 0 is 1.7\% (see first panel of Table 2). Consequently, despite the massive $4.5 \%$ decrease of world emissions in the first quarter, the average emission changes over the two-year horizon, shown in the same panel of Table 2 , is $-0.1 \%$, which is very small. This is because the decreases in emissions in advanced economies are offset by the increases in emissions in emerging economies. We next turn to the second row of Figure 3 and the second panel of Table 2, which report the change in emissions for Scenario 2 over 0. Due to the second negative shock to GDP, emissions are more negatively affected compared to Scenario 1, particularly in the second forecast year. This reduces the growth in emissions in both the advanced and the emerging economies. The average changes in the advanced and in the emerging economies are $-3.2 \%$ and $0.0 \%$, respectively. Consequently, the average world emission changes is $-1.4 \%$.

Why does our analysis forecast relatively small effects of COVID-19 on fossil fuel consumption and $\mathrm{CO}_{2}$ emissions over a two-year period? Several factors can explain the result. First, although the IMF (2020b) forecasts predict a large and immediate negative impact on GDP across countries that is unforeseen in the recent history, they indicate that the advanced economies' output recovers to the pre-pandemic level in the two-year period, while the emerging economies' output recovers even quicker. Second, existing cross-country studies on energy demand indicate that energy consumption may not be highly responsive to income changes.7 Figure 1 indicates a decline in GDP by more than $5 \%$ for the emerging economies under Scenario 1 relative to Scenario 0 in the first quarter; and more than a $10 \%$ decline for the advanced economies in the second quarter. Table 2 , on the other hand, suggests that $\mathrm{CO}_{2}$ emissions decline by a smaller magnitude in both country groups in the initial quarters. These observations are consistent with the income elasticity estimates in the literature. As the income level increases over the later quarters, the fossil fuel consumption catches up, resulting in a fast recovery of $\mathrm{CO}_{2}$ emissions.

To sum up, under Scenario 1 where the COVID-19 shock negatively affects the world economy in early 2020 but not in late 2020 to 2021, advanced economies will struggle to restore their energy consumption growth to the no COVID-19 levels until the end of 2021. In contrast, the emerging economies may recover faster from the drop in early 2020 and consume more energy than for the case without COVID-19. Consequently, total emissions in the world during 2020-2021 may not be affected much by the COVID-19 shock. However, if the world economy is further negatively affected by a second outbreak of COVID-19, then energy consumption in advanced and emerging economies will go down further. As a result, the world $\mathrm{CO}_{2}$ emissions level could be slightly less than that under the no

\footnotetext{
${ }^{6}$ We found that this simple method produces larger emission estimates compared to the BP estimates by $8.0 \%-10.1 \%$, which is in line with BP's note. See Appendix A.3 for the comparison of our estimates with BP's and related details.

${ }^{7}$ Huntington et al. (2019) reviews income elasticity estimates for liquid fuels in the literature, indicating a wide range of estimates that average at around less than 1. They find little evidence that countries with higher income levels have lower income responses.
} 
Advanced
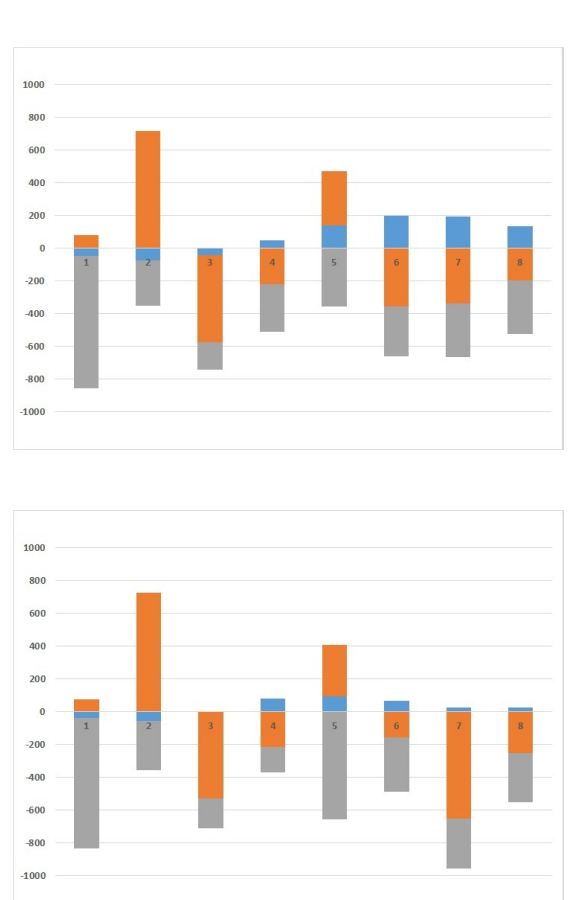

Emerging

Scenario $1-0$

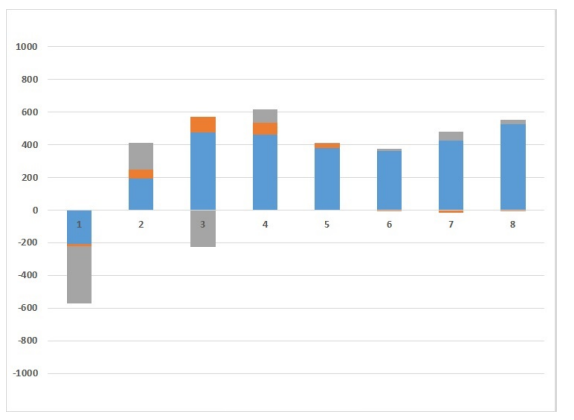

Scenario $2-0$

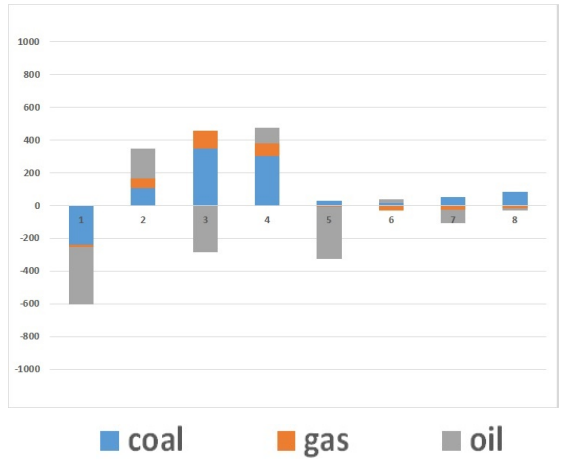

World
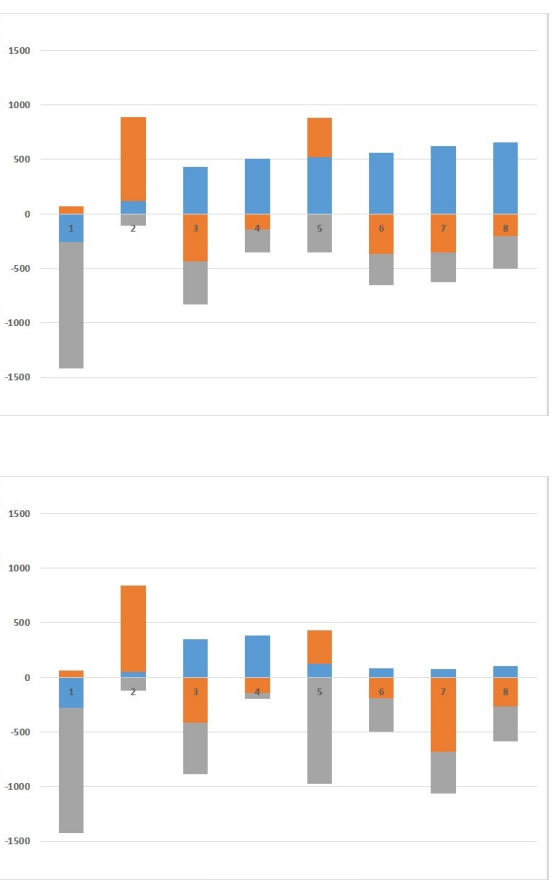

Figure 3: Difference in $\mathrm{CO}_{2}$ emissions (annualised in $\mathrm{MtCO}_{2}$ )

Table 2: Changes in $\mathrm{CO}_{2}$ emissions under Scenerios 1 and 2

\begin{tabular}{rrrrrrrrrr}
\hline & \multicolumn{4}{c}{ Scenario 1 to 0 } & & \multicolumn{4}{c}{ Scenario 2 to 0 } \\
\cline { 2 - 3 } Horizon & \multicolumn{1}{c}{ EU } & Advanced & Emerging & World & & EU & Advanced & Emerging & World \\
\hline 1 & $-18.9 \%$ & $-5.8 \%$ & $-3.5 \%$ & $-4.5 \%$ & & $-19.1 \%$ & $-5.6 \%$ & $-3.7 \%$ & $-4.6 \%$ \\
2 & $-1.1 \%$ & $2.6 \%$ & $2.4 \%$ & $2.5 \%$ & & $-0.5 \%$ & $2.6 \%$ & $2.0 \%$ & $2.3 \%$ \\
3 & $12.6 \%$ & $-5.4 \%$ & $2.0 \%$ & $-1.3 \%$ & & $15.1 \%$ & $-5.2 \%$ & $1.0 \%$ & $-1.7 \%$ \\
4 & $-1.3 \%$ & $-3.3 \%$ & $3.5 \%$ & $0.5 \%$ & & $-0.1 \%$ & $-2.0 \%$ & $2.7 \%$ & $0.6 \%$ \\
5 & $4.5 \%$ & $0.8 \%$ & $2.3 \%$ & $1.6 \%$ & & $-2.5 \%$ & $-1.8 \%$ & $-1.7 \%$ & $-1.7 \%$ \\
6 & $-6.7 \%$ & $-3.3 \%$ & $2.0 \%$ & $-0.3 \%$ & & $-9.7 \%$ & $-3.0 \%$ & $0.0 \%$ & $-1.3 \%$ \\
7 & $-5.2 \%$ & $-3.3 \%$ & $2.4 \%$ & $0.0 \%$ & & $-1.6 \%$ & $-6.7 \%$ & $-0.3 \%$ & $-3.0 \%$ \\
8 & $0.6 \%$ & $-2.8 \%$ & $2.8 \%$ & $0.5 \%$ & & $-0.3 \%$ & $-3.8 \%$ & $0.3 \%$ & $-1.4 \%$ \\
\hline Average & $-1.9 \%$ & $-2.6 \%$ & $1.7 \%$ & $-0.1 \%$ & & $-2.3 \%$ & $-3.2 \%$ & $0.0 \%$ & $-1.4 \%$ \\
\hline
\end{tabular}

\subsection{Forecasting fossil fuel prices after the COVID-19 spread}

We have seen that the global negative shock due to COVID-19 can have different impact on fuel consumption, and hence $\mathrm{CO}_{2}$ emissions, in different countries. This will result in a subsequent sharp fall in fuel prices. Our model takes into account (short-term) feedback effects of the domestic variables on the fuel prices as seen in equation (7). The conditional forecast of prices using the GDP shock scenarios are given in Figure 4. As this figure indicates (and as the evidence partially supports), under Scenario 1 the prices of coal and oil first increase then drop sharply, and exhibit a gradual recovery 

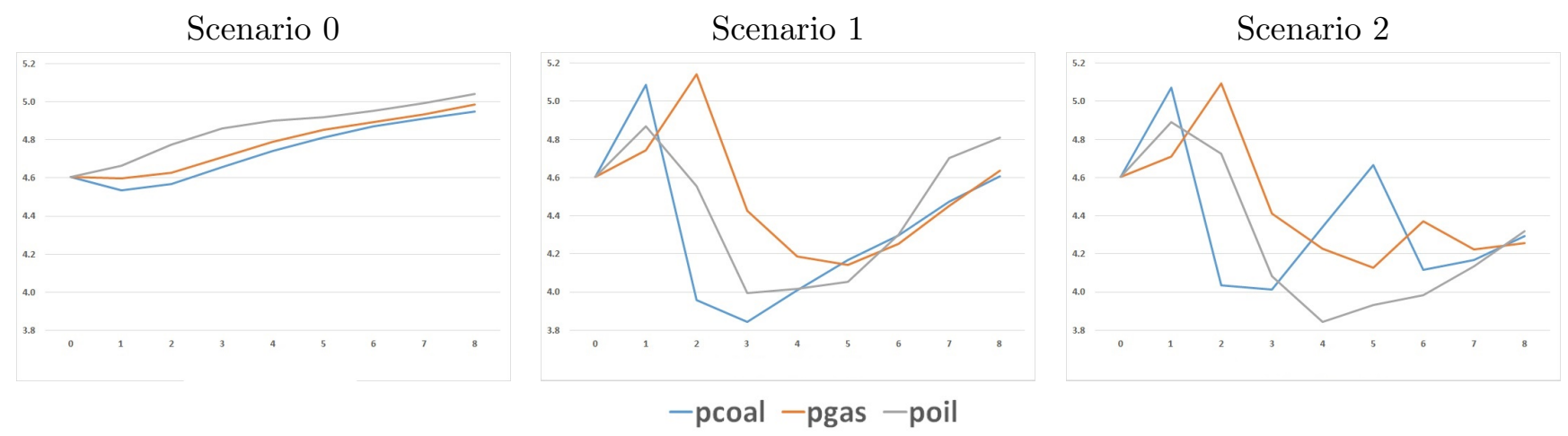

Figure 4: Conditional forecasts of fossil fuel prices conditional on GDP scenarios after the COVID-19 spread

thereafter. The drop of the natural gas price starts in the third quarter. Under Scenario 2, over the first three quarters the prices move similarly to those under Scenario 1, but from the fourth quarter onwards coal and natural gas prices exhibit a sharp rise-and-drop. Importantly, the exogenous shocks can change the relative prices of coal, natural gas and oil, which would affect the future consumption of these fuels.

\section{The effect of changes in the fossil fuel prices on fuel consumption and output}

Our analysis so far indicates that the effect of COVID-19 on the global $\mathrm{CO}_{2}$ emissions would be close to zero over the two-year time horizon though the fossil fuel prices are expected to have sizeable fluctuations (5\% or more) during this period. In particular, it shows that (i) an exogenous shock can change the relative prices of fossil fuels; and (ii) COVID-19 will not alleviate the urgency to reduce more $\mathrm{CO}_{2}$ emissions globally. In this section, we investigate the effect of changes in the relative prices of coal, natural gas and oil, due to carbon pricing, on fuel consumption and output by applying conditional forecasting to our GVAR model.

Since coal is the most carbon intensive among the three fossil fuels, we implement a counterfactual experiment that raises the coal price. We use the unconditional forecast prices as the benchmark. We can regard this experiment as one of investigating the effect of the introduction of carbon pricing to coal or the effect of a coal price increase due to market forces, or a mix of the two. For this purpose we restrict the GVAR model so that contemporaneous and lagged feedback from the domestic variables (energy consumption, GDP and exchange rates) to the fossil fuel equation are shut off and do not confound the interpretation of the experiment. Under this restriction, given the value of the prices, forecasts are produced from the following model

$$
\mathbf{x}_{t}=\hat{\mathbf{b}}_{0}+\hat{\mathbf{b}}_{1} t+\hat{\mathbf{F}}_{1} \mathbf{x}_{t-1}+\hat{\mathbf{F}}_{2} \mathbf{x}_{t-2}+\hat{\mathbf{F}}_{3} \mathbf{x}_{t-3}+\hat{\boldsymbol{\Upsilon}}_{0} \mathbf{d}_{t}+\hat{\boldsymbol{\Upsilon}}_{1} \mathbf{d}_{t-1}+\hat{\boldsymbol{\Upsilon}}_{2} \mathbf{d}_{t-2}+\hat{\mathbf{v}}_{t},
$$

where the estimated coefficients embody the internal and external linkages and dynamics resulting from the estimation of the country-specific models in Section 3.1 (for further details see Section B.2 of the Appendix). What we define as the unconditional forecast in what follows refers to the forecasts from the above model for $\mathbf{x}_{t}$, with the given global prices obtained as forecasts from the estimated $\operatorname{VAR}(3)$ representation of model (4) that includes the error-correction term given in (5). Under the counterfactual scenario, the forecasts for $\mathbf{x}_{t}$ are obtained subject to an added hike to the coal price. In order to describe long-run effects, we consider forecasts over forty quarters.

Figure 5 reports the unconditional and conditional forecasts. Each panel displays average per capita log fuel consumption and per capita log GDP for each country group. The solid lines are the unconditional forecasts and the dashed lines are conditional on (12.5\%) higher coal prices. The first panel shows that coal consumption in the advanced economies declines over the horizon, whereas it increases in the emerging economies. The second panel indicates that natural gas consumption increases worldwide, but much faster in the emerging economies. The third panel shows that oil 
Coal

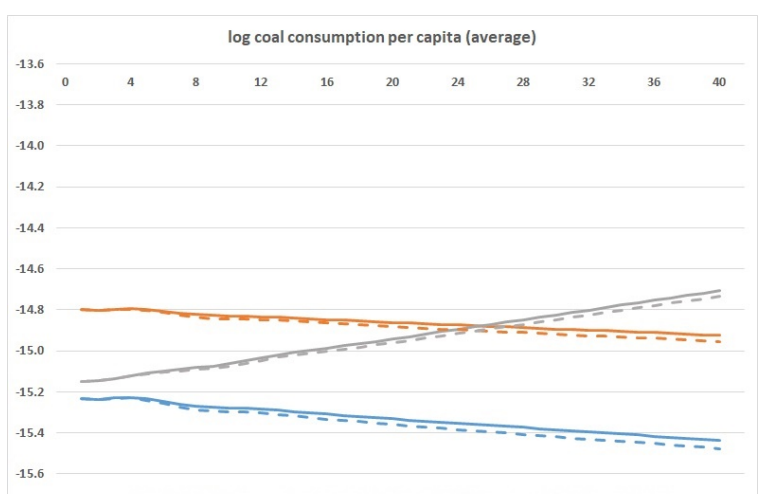

Oil

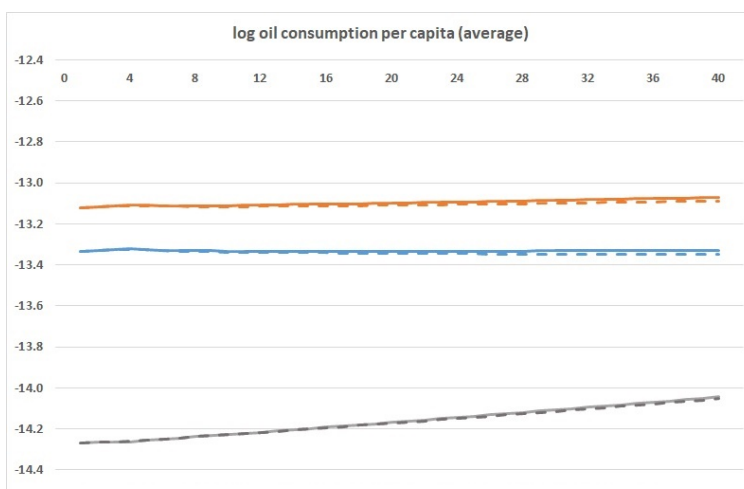

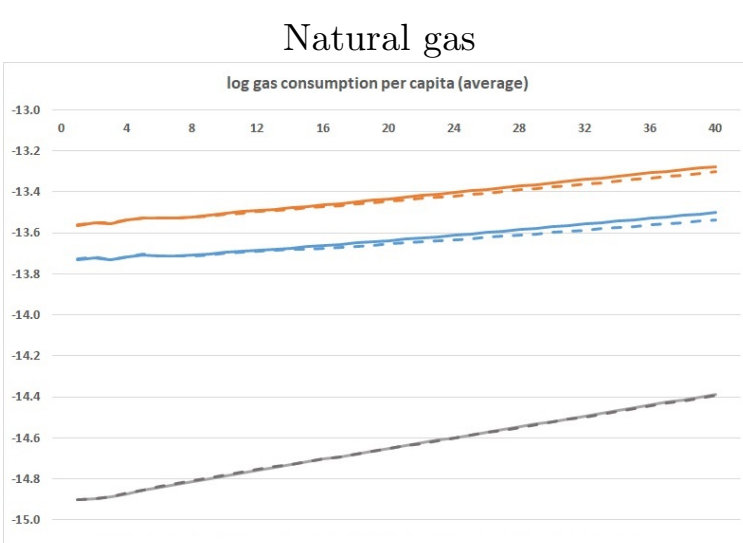

GDP

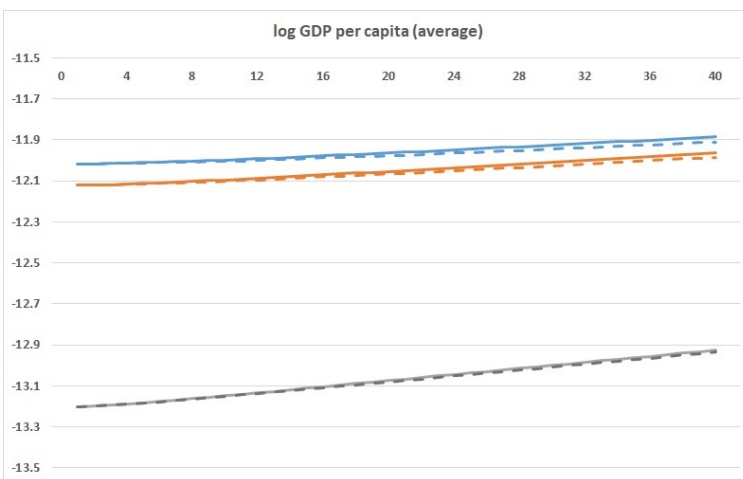

$\begin{array}{ll}\text { - EU unconditional } & \text {-Advanced unconditional - Emerging unconditional } \\ \text { - - EU conditional } & - \text { - Advanced conditional } \quad-\text { Emerging conditional }\end{array}$

Figure 5: Forecasts of fuel consumption and GDP for benchmark and higher coal price cases

$\mathrm{EU}^{+}$

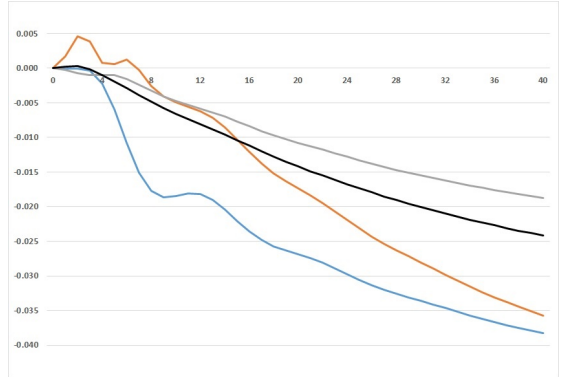

Advanced

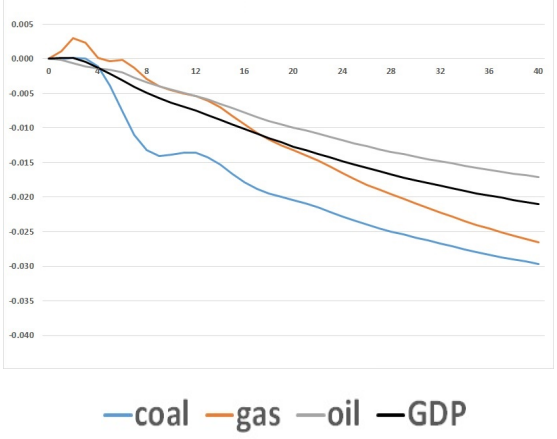

Emerging

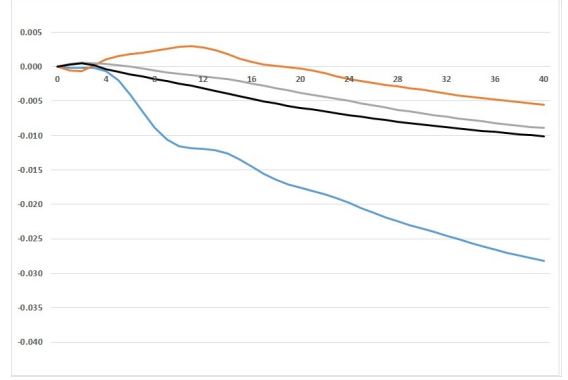

Figure 6: Difference in forecasts of fuel consumption and GDP between benchmark and higher coal price cases

consumption in the advanced economies stays almost constant over 10 years while it increases rapidly in the emerging economies. Finally, GDP grows faster in the emerging economies than in the advanced economies as illustrated in the last panel. These trajectories are remarkably similar under the scenario with higher coal prices. However, the negative effects of the higher coal price appear to be larger for $\mathrm{EU}^{+}$and the advanced economies than for the emerging ones.

In order to assess the effect of the higher coal price more clearly, we report the difference in consumption and GDP with and without the $12.5 \%$ coal price increase in Figure 6. The properties 
Table 3: The forecast elasticity of fossil fuel consumption and GDP per capita with respect to coal price

\begin{tabular}{lrrrr}
\hline & \multicolumn{4}{c}{$\mathrm{EU}^{+}$} \\
\cline { 2 - 5 } Average Annual Change & $-3.1 \%$ & $-3.1 \%$ & $-1.5 \%$ & $-2.0 \%$ \\
s.e.(autocorrelation robust) & $0.9 \%$ & $0.4 \%$ & $0.2 \%$ & $0.2 \%$ \\
t-ratio & -3.4 & -7.8 & -9.8 & -10.5 \\
p-value & 0.000 & 0.000 & 0.000 & 0.000 \\
& \multicolumn{4}{c}{ Advanced } \\
\cline { 2 - 5 } & \multicolumn{4}{c}{ coal } \\
& $-2.4 \%$ & $-2.3 \%$ & $-1.4 \%$ & $-1.7 \%$ \\
Average Annual Change & $0.7 \%$ & $0.3 \%$ & $0.1 \%$ & $0.2 \%$ \\
s.e.(autocorrelation robust) & -3.4 & -8.7 & -12.4 & -10.0 \\
t-ratio & 0.000 & 0.000 & 0.000 & 0.000 \\
p-value & \multicolumn{4}{c}{} \\
& \multicolumn{4}{c}{ Emerging } \\
\cline { 2 - 5 } & coal & gas & oil & GDP \\
Average Annual Change & $-2.3 \%$ & $-0.4 \%$ & $-0.8 \%$ & $-0.9 \%$ \\
s.e.(autocorrelation robust) & $0.5 \%$ & $0.3 \%$ & $0.1 \%$ & $0.1 \%$ \\
t-ratio & -4.8 & -1.3 & -10.1 & -9.8 \\
p-value & 0.000 & 0.091 & 0.000 & 0.000 \\
\hline
\end{tabular}

Note: The average annual change refers to the annualized average (over 40 quarters) of the quarterly change due to a $100 \%$ increase in the relative price of coal.

of the differences in consumption and GDP for the baseline and for the higher coal price are similar for $\mathrm{EU}^{+}$and the advanced economies, but the former group is more sensitive to the coal price hike. Eventually the higher coal price decreases energy consumption and GDP and the gap under the two scenarios widens. While natural gas consumption increases in the initial two years, it decreases thereafter.

Table 3 reports the 'elasticity' of fossil fuel consumption and GDP per capita due to a $100 \%$ increase in the price of coal. The elasticities are small across the board. For example, the estimated coal price elasticity of coal consumption per capita is between $-3.1 \%$ and $-2.3 \%$. This magnitude of price elasticity is within the range of estimated price elasticity of energy demand in the literature 8

For the emerging economies, the negative effect of the higher coal price on coal consumption is similar to that of the advanced economies, but the effects on GDP, oil and natural gas consumption are much smaller. In particular, the elasticity of natural gas consumption is not statistically different from zero. The results also indicate that, relative to the elasticity of coal, the negative impact of a higher coal price on GDP is smaller.

Overall, the above simulation reveals that a substantial increase in the coal price can significantly reduce fossil fuel consumption across the world; the effects on GDP are smaller than those on coal consumption, and the negative effect on GDP due to a higher coal price is more limited in the emerging than in the advanced economies.

\section{Concluding remarks}

In this paper we assess the impact of the global economic shock from COVID-19 on global fossil fuel consumption and $\mathrm{CO}_{2}$ emissions over a two-year horizon. For this purpose, we employ the global vector autoregressive (GVAR) model. The model captures cross-sectional and time series interdependencies across countries, and is thus suitable for studying the global impacts of COVID-19.

\footnotetext{
${ }^{8}$ According to a meta analysis by Huntington et al. (2019), estimated short-run price elasticity of oil demand varies substantially in the literature, averaging at -0.07 for developing countries and -0.11 for OECD countries among the reviewed studies. Note that our estimate is not the price elasticity of energy demand, so it is not directly comparable to the elasticity estimates in the literature.
} 
The analysis based on data for 32 countries, which generate $81 \%$ of the global $\mathrm{CO}_{2}$ emissions due to fossil fuel use, indicates that the negative effect of COVID-19 on global fossil fuel consumption, and hence $\mathrm{CO}_{2}$ emissions, is large in the first quarter but limited over the two-year horizon. The COVID19 shock negatively affects the world economy in the first quarter after the pandemic occurs, but it is expected to recover in about a year. The advanced economies will struggle to restore their energy consumption growth by the end of the two years to the level under the counterfactual scenario without COVID-19. On the other hand, the emerging economies may recover from the drop in early 2020 rather quickly and consume more energy than for the case of no COVID-19 outbreak. Consequently, total emissions in the world during 2020-2021 may not be affected much by the COVID-19 shock. However, if the world economy is further negatively affected, such as by a second wave of the coronavirus, energy consumption in advanced and emerging economies will further go down, and emissions could be much less than in the case of no COVID-19 shock.

What will happen if these economies adopt carbon pricing which increases the relative price of coal? Our conditional forecast analysis reveals that higher coal prices lead to lower fossil fuel consumption with less-than-proportional decreases in GDP. In particular, the impact on fossil fuel consumption and GDP is smaller for the emerging economies.

Overall, our GVAR analysis indicates that the pandemic and the resulting economic shut down will not lead to a sizeable reduction in $\mathrm{CO}_{2}$ emissions over a two-year time horizon. Thus COVID-19 would not provide countries with a reason to delay climate-change mitigation efforts. Fossil fuel price increases due to carbon pricing will slow down GDP growth. However, our conditional forecast analysis with a higher coal price demonstrates that the effect on GDP is limited compared to its impact on fossil fuel consumption.

Due to lower output under the pandemic and scaled-down industrial activities, the EUETS Allowance price dropped sharply in mid March 2020 though in the last three months since then it has been increasing to recover to the pre-crisis level. Our analysis implies that continued efforts to reduce $\mathrm{CO}_{2}$ emissions, through an expanded introduction of carbon pricing in other countries, may not be too costly in terms of the size of the GDP impacts. As the effects on GDP are expected to be more limited in the emerging than in advanced economies, global application of carbon pricing may not lead to concerning distributional impacts across countries with different income levels.

With regard to the impact of the economic shock from COVID-19 on the advanced versus emerging economies, which is revealed by our analysis, the following caveats are in order: our analysis does not study the effects on each country separately; stringent financing restrictions in emerging economies may drag down their recovery from the pandemic's negative economic effects (IMF, 2020b). Considering a global model that can reflect differences in sovereign credits across countries is left for future research.

Our analysis also demonstrates differential impacts of slower GDP growth due to COVID-19 on different fossil fuel sources in different parts of the world. The simulation associated with a higher coal price also indicated different effects on natural gas and oil consumption. In the context of climate change mitigation, it would be useful to consider the impact on renewable energy integration, which this paper does not address due to data availability. Future research could address how COVID-19's negative economic shocks influence the speed of renewables integration.

\section{Appendix A: Further Description of the Data}

\section{A.1 Data Sources}

\section{A.1.1 Macroeconomic variables, PPP-GDP and trade data}

Real GDP and the real exchange rate for all countries, as well as PPP-GDP figures and the trade data for construction of the trade matrix (used in computing the foreign variables of the GVAR model) were taken from the GVAR 2016 Vintage available at https://sites.google.com/site/gvarmodelling/data. This is an updated version of the 2013 Vintage, updated by Kamiar Mohaddes and Mehdi Raissi. The PPP-GDP data are from the World Development Indicator database of the World Bank. The trade data is from the IMF Direction of Trade statistics constructed based on the average of Exports and Imports (c.i.f.) at the annual frequency. Further details including the source of the macroeconomic variables for each country can be found there. 


\section{A.1.2 Population}

Population data are from the World Bank website https://data.worldbank.org/indicator/SP.POP.TOTL available at the annual frequency. The annual data were interpolated to obtain the quarterly values using the approx function in R selecting the method "linear."

\section{A.1.3 Energy consumption}

Energy consumption data were obtained from Oxford Economics (https://www.oxfordeconomics.com/) whose provider is the International Energy Agency (IEA). The Lisman and Sandee (1964) method was used for interpolation where required.

Data for coal consumption (domestic demand, annualised, Mtoe) for all countries are constructed from the IEA World Energy Balances service, Summary and Extended Energy Balances database which contains annual data. Quarterly values were interpolated from the annual series.

Data for natural gas consumption (domestic demand, annualised, Mtoe) for all OECD countries are constructed from the IEA Natural Gas Monthly service, Natural Gas Balance database. Monthly figures were summed to obtain the quarterly values. Quarterly data for non-OECD countries were interpolated from annual data which were constructed from the IEA World Energy Balances service, Summary and Extended Energy Balances database.

Data for oil consumption (domestic demand, annualised, Mtoe) for all OECD countries, and nonOECD countries over the period 1991-2014, are from the IEA Monthly Oil Service. Monthly figures are summed to obtain the quarterly data. Quarterly values for non-OECD data for the period 1991Q12014Q4 were interpolated from the annual data of the IEA World Energy Balances service.

\section{A.1.4 Global energy prices}

The coal, natural gas and oil quarterly prices are computed from the monthly prices obtained from the World Bank Commodity Price Data ('Pink Sheet' Data).

\section{A.1.5 Temperature data}

Daily temperature data from monitoring stations across the countries of interest were extracted from the Global Historical Climatology Network- Daily (GHCN) hosted by the National Centers for Environmental Information (NCEI) of the National Oceanic and Atmospheric Administration (NOAA) over the period 1981-2014. GHCN daily is an integrated database of daily climate summaries from land surface stations across the globe that have been subjected to a common suite of quality assurance reviews. It contains records from over 100,000 stations in 180 countries and territories. NCEI provides numerous daily variables, including maximum temperature (Tmin) and minimum temperature (Tmax), total daily precipitation, snowfall, and snow depth. Both the record length and period of record vary by station and cover intervals ranging from less than a year to more than 175 years.

For each monitoring station, all available data that fulfilled the following criteria were retained: (i) both Tmax and Tmin were available for each day and (ii) no quality assurance or quality control issues were identified for either Tmax or Tmin. For those countries with missing data over the period of interest, namely Canada, Germany, Singapore and UK, data from the monitoring stations of the top three largest metropolitan areas based on population were included. Similarly for the US, data from the monitoring stations of the top three largest states in terms of population were included namely California, Texas and New York.

\section{A.2 Temperature and Seasonal Adjustment}

\section{A.2.1 Construction of HDD and CDD}

The daily temperature data were converted from degree Fahrenheit to Celsius and heating and cooling degree days, $H D D_{i \tau d}$ and $C D D_{i \tau d}$ respectively, were construted for each country $i$ and each day $d$ of 
each year $\tau$ as follows

$$
\begin{aligned}
& H D D_{i \tau d}=\left\{\begin{array}{c}
18{ }^{\circ} \mathrm{C}-\text { mean daily temperature of country } i \\
0, \quad \text { if } 18{ }^{\circ} \mathrm{C}>\text { mean daily temperature of country } i
\end{array}\right. \\
& C D D_{i \tau d}=\left\{\begin{array}{c}
\text { mean daily temperature of country } i-21{ }^{\circ} \mathrm{C} \\
0, \quad \text { if } 21^{\circ} \mathrm{C}<\text { mean daily temperature of country } i
\end{array}\right.
\end{aligned}
$$

where mean daily temperature was computed as $(\mathrm{Tmin}+\mathrm{Tmin}) / 2$.

The corresponding quarterly data for $H D D_{i t}$ and $C D D_{i t}$ for $t=1,2, \ldots, T$ were constructed by taking the average daily $H D D_{i \tau d}$ and $C D D_{i \tau d}$ values for each of the four quarters. Missing values of no more than six consecutive values were encountered for HDD for Germany and for CDD for Germany, Indonesia and Malaysia. These gaps were filled using a simple linear interpolation method.

\section{A.2.2 Temperature adjustment}

Let $y_{i t}$ be quarterly energy consumption for country $i$. Following common practice (see for example Elkhafif, 1996) temperature adjustment of energy consumption was performed as follows:

1. Run the regression based on the quarterly data

$$
y_{i t}=b_{0 i}+b_{1 i} H D D_{i t}+b_{2 i} C D D_{i t}+\varepsilon_{i t}, t=1,2, \ldots, T .
$$

Equation (A.1) may consist of only $H D D_{i t}$ or $C D D_{i t}$ see Table A.2.

2. Construct the correction factor as

$$
C O R R_{i \tau q}=\hat{b}_{1 i}\left(H D D_{i \tau q}-N H D D_{i q}\right)+\hat{b}_{2 i}\left(C D D_{i \tau q}-N C D D_{i q}\right)
$$

where $\tau$ is the year $(\tau=1984,1985, \ldots, 2014)$ and $q$ is the quarter $(q=Q 1, Q 2, Q 3, Q 4)$,

$$
\begin{aligned}
& N H D D_{i q}=\text { the } 30 \text { year average of } H D D_{i} \text { for the } q^{\text {th }} \text { quarter }(1981-2010) \\
& N C D D_{i q}=\text { the } 30 \text { year average of } C D D_{i} \text { for the } q^{\text {th }} \text { quarter (1981-2010), }
\end{aligned}
$$

are climate normals, typically computed over a three consecutive ten-year period the most recent being 1981-2010, see Won et al. (2016) for a discussion of climate normals.

3. The temperature adjusted consumption series is then

$$
\left(y_{i t}\right)_{t a}=y_{i t}-C O R R_{i \tau q} .
$$

\section{A.2.3 Seasonal adjustment}

Joint significance of seasonal components was tested for all consumption and price series based on the procedure described in Section A.2.4. For consumption, seasonal effects were not significant for any of the annual series namely all coal series and the natural gas non-OECD series. Significant seasonal effects were found for all natural gas OECD series with the exception of Norway and for all oil series with the exception of Indonesia $2^{9}$ and Malaysia. No temperature and/or seasonal adjustment was performed for the natural gas series of Norway and the oil series of Malaysia nor for the coal and non-OECD natural gas series. For prices, only the natural gas prices exhibited seasonal effects and was seasonally adjusted.

To seasonally adjust the data we start from $\log (y)$ (where $y$ here is either the original consumption series or the temperature adjusted one, or the natural gas price series) and take the first difference, $\Delta \log (y)$. This is seasonally adjusted using the X-12 quarterly seasonal adjustment method under the additive option to obtain $\Delta \log (y)_{s a}$. Then using the first observation of the raw series $\log (y)(\operatorname{levels,}$ not seasonally adjusted) the seasonally adjusted log changes, $\Delta \log (y)_{s a}$, are cumulated to obtain the $\log$ adjusted series $\log (y)_{s a}$. Finally, the seasonal adjusted level series, $(y)_{s a}$, is obtained by taking the exponential of $\log (y)_{s a}$.

\footnotetext{
${ }^{9}$ For the oil series of Indonesia we did peform seasonal adjustent despite the non-significant finding of the seasonality test as the high volatility of the series appeared to obscure the clear seasonal pattern in the less volatile part of the series
} 
Table A.2 summarises all adjustments performed to the energy consumption series for each country. It also includes information on the available HDD and CDD series for each country, with a 'no' indicating that the corresponding series was zero throughout the sample period 1984-2014 and was therefore not included in the temperature adjustment procedure (if performed), yes indicating otherwise.

Table A.2: Summary of adjustments to energy consumption of each country

\begin{tabular}{|c|c|c|c|c|c|}
\hline & Coal & Natural Gas & Oil & HDD & CDD \\
\hline Argentina & original data & original data & sa & yes & yes \\
\hline Australia & original data & ta \& sa & ta \& sa & yes & yes \\
\hline Austria & original data & ta \& sa & $\mathrm{sa}$ & yes & yes \\
\hline Belgium & original data & ta \& sa & sa & yes & yes \\
\hline Brazil & original data & original data & $\mathrm{sa}$ & yes & yes \\
\hline Canada & original data & sa & sa & yes & yes \\
\hline Chile & original data & $\mathrm{sa}$ & sa & yes & yes \\
\hline China & original data & original data & sa & yes & yes \\
\hline Finland & original data & ta \& sa & ta \& sa & yes & no \\
\hline France & original data & ta \& sa & sa & yes & yes \\
\hline Germany & original data & sa & ta \& sa & yes & yes \\
\hline India & original data & original data & sa & yes & yes \\
\hline Indonesia & original data & original data & $\mathrm{sa}$ & no & yes \\
\hline Italy & original data & sa & ta \& sa & yes & yes \\
\hline Japan & original data & sa & ta \& sa & yes & yes \\
\hline Korea & original data & - & $\mathrm{sa}$ & yes & yes \\
\hline Malaysia & original data & original data & original data & no & yes \\
\hline Mexico & original data & $\mathrm{sa}$ & $\mathrm{sa}$ & yes & yes \\
\hline Netherlands & original data & sa & sa & yes & yes \\
\hline New Zealand & original data & ta \& sa & sa & yes & yes \\
\hline Norway & original data & original data & $\mathrm{sa}$ & yes & no \\
\hline Philippines & original data & - & $\mathrm{sa}$ & no & yes \\
\hline Saudi Arabia & - & original data & $\mathrm{sa}$ & yes & yes \\
\hline Singapore & - & original data & sa & no & yes \\
\hline South Africa & original data & - & sa & yes & yes \\
\hline Spain & original data & sa & ta \& sa & yes & yes \\
\hline Sweden & original data & - & ta \& sa & yes & no \\
\hline Switzerland & original data & ta \& sa & sa & yes & yes \\
\hline Thailand & original data & original data & sa & no & yes \\
\hline Turkey & original data & - & sa & yes & yes \\
\hline UK & original data & sa & ta \& sa & yes & yes \\
\hline US & original data & ta \& sa & ta \& sa & yes & yes \\
\hline
\end{tabular}

Note: 'original data' signifies that the no temperature and/or seasonal adjustment was performed on the corresponding series. 'ta' and 'sa' signifies temperature adjusted and seasonally adjusted respectively. For the oil series any 'ta' and/or 'sa' for non-OECD countries was performed over the period 1991Q1-2014Q4 as prior to 1991 the series are interpolated from annual data. 'no' for any HDD or CDD series signifies that all values for the corresponding series were zero throughout the sample for the associated country and was therefore not included in the temperature adjustment procedure (if performed), 'yes' signifies otherwise.

\section{A.2.4 Assessing the joint significance of seasonal effects}

To assess the joint significance of the seasonal components for a series $y$ we consider its natural logarithm denoted by $\log (y)$, and use the following procedure:

1. Let $S_{1}, S_{2}, S_{3}$ and $S_{4}$ be the usual seasonal dummies, such that $S_{i}, i=1,2,3,4$, takes the value of 1 in the $i^{\text {th }}$ quarter and zero in the remaining three quarters.

2. Construct $S_{14}=S_{1}-S_{4}, S_{24}=S_{2}-S_{4}, S_{34}=S_{3}-S_{4}$.

3. Run a regression of $\Delta \log (y)$ (where the lower case denotes the natural logarithm of the corresponding variable) on an intercept and $S_{14}, S_{24}, S_{34}$. Denote the OLS estimates of $S_{14}, S_{24}$ and $S_{34}$ by $a_{1}, a_{2}$ and $a_{3}$. 
4. Asses the joint significance of the seasonal components by testing the hypothesis that $a_{1}=a_{2}=$ $a_{3}=0$ using the F-statistic.

5. In cases where the null hypothesis was rejected at the $10 \%$ level, seasonal adjustment was performed on the log-difference of the original series using the $\mathrm{X}-12$ procedure as described above.

\section{A.3 Comparison of Consumption and Emissions Data with BP Data}

Our $\mathrm{CO}_{2}$ emissions were calculated by applying the single emission factor used by $\mathrm{BP}^{10}$ to each of the coal, natural gas and oil series. These emission factors are based on standard global average conversion factors compiled on the basis of average carbon content: coal at 94,600 $\mathrm{kg} \mathrm{CO}_{2}$ per TJ (3.96 tonnes per tonne of oil equivalent); natural gas at 56,100 $\mathrm{kg} \mathrm{CO}_{2}$ per TJ (2.35 tonnes per tonne of oil equivalent); and oil at 73,300 $\mathrm{kg} \mathrm{CO}$ per TJ (3.07 tonnes per tonne of oil equivalent).

We compared our $\mathrm{CO}_{2}$ emission data with published BP data, bp-stats-review-2019-consolidated -dataset-panel-format.xlsx. The comparison is based on 2014Q4 annualised consumption and the $2014 \mathrm{BP}$ data file ${ }^{11}$ for the four country groups of Table 1: (i) $\mathrm{EU}^{+}$, (ii) Advanced, (iii) Emerging, (iv) Total. The $\mathrm{CO}_{2}$ emissions and consumption of coal, natural gas and oil per Mtoe is given in the table that follows.

Table A.3: $\mathrm{CO}_{2}$ emissions and consumption of coal, natural gas and oil per Mtoe
(a) Our consumption
(b) BP consumption
(c) Emission Factors
(d) $\mathrm{CO}_{2}(\mathrm{a}) \times(\mathrm{c})$
(e) $\mathrm{CO}_{2}(\mathrm{~b}) \times(\mathrm{c})$
(f) $\mathrm{BP} \mathrm{CO} 2$
(g) $\log ((\mathrm{d}) /(\mathrm{e}))(\%)$
(h) $\log ((\mathrm{e}) /(\mathrm{f}))(\%)$
(i) $\log ((\mathrm{d}) /(\mathrm{f}))(\%)$

\begin{tabular}{|c|c|c|c|}
\hline \multicolumn{4}{|c|}{ (i) Advanced } \\
\hline coal & gas & oil & total \\
\hline 851.1 & 1725.1 & 2108.9 & 4685.1 \\
\hline 866.5 & 1201.9 & 1935.7 & 4004.1 \\
\hline 3.96 & 2.35 & 3.07 & \\
\hline 3370.2 & 4054.1 & 6474.4 & 13898.6 \\
\hline 3431.3 & 2824.5 & 5942.6 & 12198.3 \\
\hline & & & 11023.9 \\
\hline$-1.8 \%$ & $36.1 \%$ & $8.6 \%$ & $13.0 \%$ \\
\hline & & & $10.1 \%$ \\
\hline & & & $23.2 \%$ \\
\hline
\end{tabular}

\begin{tabular}{rrrrr}
\multicolumn{4}{c}{ (ii) Emerging } & \\
\cline { 1 - 3 } coal & \multicolumn{1}{c}{ gas } & \multicolumn{1}{l}{ oil } & & total \\
\hline 2683.7 & 533.4 & 1472.5 & & 4689.6 \\
2596.0 & 597.4 & 1431.3 & & 4624.8 \\
3.96 & 2.35 & 3.07 & \\
10627.5 & 1253.4 & 4520.7 & & 16401.6 \\
10280.2 & 1403.9 & 4394.2 & & 16078.4 \\
& & & & 14988.2 \\
$3.3 \%$ & $-11.3 \%$ & $2.8 \%$ & & $2.0 \%$ \\
& & & \\
& & & \\
& & & \\
\end{tabular}

\begin{tabular}{|c|c|c|c|c|c|c|c|c|}
\hline \multirow[b]{3}{*}{ (a) Our consumption } & \multicolumn{4}{|c|}{ (iii) $\mathrm{EU}^{+}$} & \multicolumn{4}{|c|}{ Total (i) + (ii) } \\
\hline & coal & gas & oil & total & coal & gas & oil & total \\
\hline & 164.3 & 480.5 & 553.5 & 1198.3 & 3534.8 & 2258.5 & 3581.5 & \\
\hline (b) $\mathrm{BP}$ consumption & 165.7 & 288.5 & 517.4 & 971.6 & 3462.5 & 1799.3 & 3367.0 & 8628.8 \\
\hline (c) Emission Factors & 3.96 & 2.35 & 3.07 & & 3.96 & 2.35 & 3.07 & \\
\hline (d) $\mathrm{CO}_{2}(\mathrm{a}) \times(\mathrm{c})$ & 650.8 & 1129.2 & 1699.2 & 3479.1 & 13997.7 & 5307.5 & 10995.1 & 30300.2 \\
\hline (e) $\mathrm{CO}_{2}(\mathrm{~b}) \times(\mathrm{c})$ & 656.2 & 677.9 & 1588.5 & 2922.6 & 13711.5 & 4228.4 & 10336.8 & 28276.7 \\
\hline (f) $\mathrm{BP} \mathrm{CO}_{2}$ & & & & 2651.6 & & & & 26012.1 \\
\hline$(\mathrm{g}) \log ((\mathrm{d}) /(\mathrm{e}))(\%)$ & $-0.8 \%$ & $51.0 \%$ & $6.7 \%$ & $17.4 \%$ & $2.1 \%$ & $22.7 \%$ & $6.2 \%$ & $6.9 \%$ \\
\hline (h) $\log ((\mathrm{e}) /(\mathrm{f}))(\%)$ & & & & $9.7 \%$ & & & & $8.3 \%$ \\
\hline (i) $\log ((\mathrm{d}) /(\mathrm{f}))(\%)$ & & & & $27.2 \%$ & & & & $15.3 \%$ \\
\hline
\end{tabular}

Note: The difference between our $\mathrm{CO}_{2}$ emission estimates (d) and those of BP (f) for total emissions is $15.3 \%$. This discrepancy is wider for advanced economies (23.2\%) than for emerging economies (9.0\%). This difference can be decomposed into two parts: that from converting energy consumption into emissions ( $h$ ) and that associated with the calculation of consumption (g). Our simple conversion method using the factor (c) tends to overestimate emissions by $7.0 \%-10.1 \%$, which is in line with BP's note according to footnote 11 below. The other source of discrepancy related to consumption calculation is negligible for coal and moderate for oil, but substantial for natural gas. The difference between our natural gas consumption figures (a) and those of BP (b) are reported in (g) as 36.1\%, -11.3\%, and 22.7\%

\footnotetext{
${ }^{10}$ These are the emission factors that BP used to estimate carbon emissions from energy consumption prior to revising their process for the 2016 edition of the Statistical Review.2016.

${ }^{11}$ While these figures are based on BP's revised methodology for constructing $\mathrm{CO}_{2}$ emissions since 2016 , it is mentioned in their note that applying their emission factors we use here would result in $\mathrm{CO}_{2}$ emissions about $8 \%$ higher than those derived from their revised methodology.
} 
for the Advanced, Emerging and Total groups, respectively. Since natural gas is far more used by the advanced group, the discrepancy in emission estimates due to the difference in energy consumption is larger for this group: these (g) are $13 \%, 2.0 \%$ and $6.9 \%$ for advanced, emerging and total, respectively.

\section{Appendix B: Further Description and Results of the GVAR Model}

\section{B.1 Country-specific Variables}

Table B.1(i) Summary of domestic and foreign variables included in the country-specific models

\begin{tabular}{|l|c|c|c|c|}
\hline \multicolumn{2}{|c|}{ All Countries Excluding US } & \multicolumn{2}{c|}{ US } \\
\hline Variables & Endogenous & Weakly Exogenous & Endogenous & Weakly Exogenous \\
\hline GDP per capita & $g d p_{i t}$ & $g d p_{i t}^{*}$ & $g d p_{U S, t}$ & gd $p_{U S, t}^{*}$ \\
\hline Coal consumption & coal & coal $_{i t}^{*}$ & coal $_{U S, t}$ & coal $_{U S, t}^{*}$ \\
Nat. gas consumption & $g a s_{i t}$ & $g a s_{i t}^{*}$ & $g a s_{U S, t}$ & gas $_{U S, t}^{*}$ \\
Oil consumption & oil $_{i t}$ & oil $_{i t}^{*}$ & oil $_{U S, t}$ & oil $_{U S, t}^{*}$ \\
Real exchange rate & $e p_{i t}$ & - & - & ep $_{U S, t}^{*}$ \\
Coal price & - & pcoal $_{t}$ & - & pcoal $_{t}$ \\
Nat. gas price & - & pgast $_{t}$ & - & pgas $_{t}$ \\
Oil price & - & poil $_{t}$ & - & poil $_{t}$ \\
\hline
\end{tabular}

Table B.1(ii) Composition of country variables

\begin{tabular}{|lcl|}
\hline Variables & \# Countries & \\
\hline GDP per capita & 32 & \\
\hline Real exchange rate & 31 & Excluding: US \\
\hline Coal consumption & 30 & Excluding: Saudi Arabia and Singapore \\
\hline Nat. gas consumption & 27 & $\begin{array}{l}\text { Excluding: Korea, Philippines, South Africa, } \\
\text { Sweden, Turkey }\end{array}$ \\
\hline Oil consumption & 32 & \\
\hline
\end{tabular}

Note: The excluded consumption series had at least some part of the series equal to zero.

\section{B.2 Solving for the GVAR model}

We solve for the GVAR model in terms of $\mathbf{y}_{t}=\left(\mathbf{x}_{t}^{\prime}, \mathbf{d}_{t}^{\prime}\right)^{\prime}$, using the the estimated VECMX* models (2) and the global price model (7). We initially obtain the global model associated with the individual country equations given by (2), expressed in terms of the $k \times 1$ global variable vector $\mathbf{x}_{t}=\left(\mathbf{x}_{0 t}^{\prime}, \mathbf{x}_{1 t}^{\prime}, \ldots, \mathbf{x}_{N t}^{\prime}\right)^{\prime}$ with $k=\sum_{i=0}^{N} k_{i}$. To this end, by setting $\mathbf{z}_{i t}=\left(\mathbf{x}_{i t}^{\prime}, \mathbf{x}_{i t}^{* \prime}\right)^{\prime}(2)$ can be expressed in terms of $\mathbf{z}_{i t}$ as follows

$$
\begin{aligned}
\mathbf{G}_{i 0} \mathbf{z}_{i t} & =\mathbf{a}_{i 0}+\mathbf{a}_{i 1} t+\mathbf{G}_{i 1} \mathbf{z}_{i, t-1}+\ldots+\mathbf{G}_{i p_{i}} \mathbf{z}_{i, t-p_{i}} \\
& +\mathbf{\Psi}_{i 1} \mathbf{d}_{t-1}+\ldots+\mathbf{\Psi}_{i q_{i}} \mathbf{d}_{t-q_{i}}+\mathbf{u}_{i t},
\end{aligned}
$$

where $\mathbf{G}_{i 0}=\left(\mathbf{I}_{k_{i}},-\boldsymbol{\Lambda}_{i 0}\right)$ and $\mathbf{G}_{i j}=\left(\mathbf{\Phi}_{i j}, \boldsymbol{\Lambda}_{i j}\right)$, for $j=1, \ldots, p_{i}$. Then using the identity $\mathbf{z}_{i t}=\mathbf{W}_{i} \mathbf{x}_{t}$, for $i=0,1, \ldots, N$, where $\mathbf{W}_{i}$ are the link matrices defined by the trade weights $w_{i j}$, (B.1) can be written as

$$
\begin{aligned}
\mathbf{G}_{i 0} \mathbf{W}_{i} \mathbf{x}_{t} & =\mathbf{a}_{i 0}+\mathbf{a}_{i 1} t+\mathbf{G}_{i 1} \mathbf{W}_{i} \mathbf{x}_{t-1}+\ldots+\mathbf{G}_{i p_{i}} \mathbf{W}_{i} \mathbf{x}_{t-p_{i}}+\mathbf{\Psi}_{i 0} \mathbf{d}_{t}+ \\
& +\mathbf{\Psi}_{i 1} \mathbf{d}_{t-1}+\ldots+\mathbf{\Psi}_{i q_{i}} \mathbf{d}_{t-q_{i}}+\mathbf{u}_{i t} .
\end{aligned}
$$

The individual models in (B.2) are then stacked to yield the model for $\mathbf{x}_{t}$ given by

$$
\mathbf{G}_{0} \mathbf{x}_{t}=\mathbf{a}_{0}+\mathbf{a}_{1} t+\mathbf{G}_{1} \mathbf{x}_{t-1}+\ldots+\mathbf{G}_{p} \mathbf{x}_{t-p}+\mathbf{\Psi}_{0} \mathbf{d}_{t}+\mathbf{\Psi}_{1} \mathbf{d}_{t-1}+\ldots+\mathbf{\Psi}_{q} \mathbf{d}_{t-q}+\mathbf{u}_{t}
$$


where $p=\max \left(p_{i}\right), q=\max \left(q_{i}\right)$, and

$$
\begin{gathered}
\mathbf{G}_{j}=\left(\begin{array}{c}
\mathbf{G}_{0 j} \mathbf{W}_{0} \\
\mathbf{G}_{1 j} \mathbf{W}_{1} \\
\vdots \\
\mathbf{G}_{N j} \mathbf{W}_{N}
\end{array}\right), \mathbf{\Psi}_{s}=\left(\begin{array}{c}
\mathbf{\Psi}_{0 s} \\
\boldsymbol{\Psi}_{1 s} \\
\vdots \\
\boldsymbol{\Psi}_{N s}
\end{array}\right), \begin{array}{c}
j=0,1, \ldots, p ; \\
s=0,1, \ldots, q,
\end{array} \\
\mathbf{a}_{0}=\left(\begin{array}{c}
\mathbf{a}_{00} \\
\mathbf{a}_{10} \\
\vdots \\
\mathbf{a}_{N 0}
\end{array}\right), \mathbf{a}_{1}=\left(\begin{array}{c}
\mathbf{a}_{01} \\
\mathbf{a}_{11} \\
\vdots \\
\mathbf{a}_{N 1}
\end{array}\right), \mathbf{u}_{t}=\left(\begin{array}{c}
\mathbf{u}_{0 t} \\
\mathbf{u}_{1 t} \\
\vdots \\
\mathbf{u}_{N t}
\end{array}\right) .
\end{gathered}
$$

Setting $\widetilde{\mathbf{x}}_{t}=\sum_{i=0}^{N} \widetilde{w}_{i} \mathbf{x}_{i t}=\widetilde{\mathbf{W}} \mathbf{x}_{t},{ }^{12}$ and assuming that $p=\widetilde{p}=\widetilde{q}=q$ for ease of exposition, (B.3) and (7) can be written in terms of $\mathbf{y}_{t}=\left(\mathbf{x}_{t}^{\prime}, \mathbf{d}_{t}^{\prime}\right)^{\prime}$ as

$$
\mathbf{H}_{0} \mathbf{y}_{t}=\mathbf{h}_{0}+\mathbf{h}_{1} t+\mathbf{H}_{1} \mathbf{y}_{t-1}+\ldots+\mathbf{H}_{p} \mathbf{y}_{t-p}+\boldsymbol{\zeta}_{t}
$$

where

$$
\begin{aligned}
& \mathbf{H}_{0}=\left[\begin{array}{cc}
\mathbf{G}_{0} & -\mathbf{\Psi}_{0} \\
\mathbf{0}_{m_{d} \times k} & \mathbf{I}_{m_{d}}
\end{array}\right], \mathbf{h}_{0}=\left[\begin{array}{l}
\mathbf{a}_{0} \\
\boldsymbol{\mu}_{0}
\end{array}\right], \mathbf{h}_{1}=\left[\begin{array}{l}
\mathbf{a}_{1} \\
\boldsymbol{\mu}_{1}
\end{array}\right], \\
& \mathbf{H}_{j}=\left[\begin{array}{cc}
\mathbf{G}_{j} & \boldsymbol{\Psi}_{j} \\
\boldsymbol{\Lambda}_{x j} \widetilde{\mathbf{W}}_{j} & \boldsymbol{\Phi}_{d j}
\end{array}\right], j=1, \ldots, p, \quad \boldsymbol{\zeta}_{t}=\left[\begin{array}{l}
\mathbf{u}_{t} \\
\boldsymbol{\eta}_{t}
\end{array}\right] .
\end{aligned}
$$

Assuming that $\mathbf{H}_{0}$ is invertible, premultiplying (B.4) by $\mathbf{H}_{0}^{-1}$ we arrive at the GVAR model

$$
\mathbf{y}_{t}=\mathbf{c}_{0}+\mathbf{c}_{1} t+\mathbf{C}_{1} \mathbf{y}_{t-1}+\ldots+\mathbf{C}_{p} \mathbf{y}_{t-p}+\boldsymbol{\varepsilon}_{t},
$$

with $\mathbf{c}_{j}=\mathbf{H}_{0}^{-1} \mathbf{h}_{j}, j=0,1, \mathbf{C}_{j}=\mathbf{H}_{0}^{-1} \mathbf{H}_{j}, j=1, \ldots, p$, and $\varepsilon_{t}=\mathbf{H}_{0}^{-1} \boldsymbol{\zeta}_{t}$, which is the model used for forecasting. In general, the lag order of $\mathbf{y}_{t}$ will be determined by the maximum lag order $\max (\max (p, \widetilde{p}), \max (q, \widetilde{q}))$. In our case the final estimated GVAR is of order 3. Related output on the stability of model (B.5) can be found in the supplementary material. ${ }^{13}$

For the purpose of forecasting when the global prices are taken as given (and no feedback effects

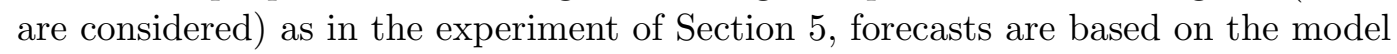

$$
\mathbf{x}_{t}=\mathbf{b}_{0}+\mathbf{b}_{1} t+\mathbf{F}_{1} \mathbf{x}_{t-1}+\ldots+\mathbf{F}_{p} \mathbf{x}_{t-p}+\mathbf{\Upsilon}_{0} \mathbf{d}_{t}+\mathbf{\Upsilon}_{1} \mathbf{d}_{t-1}+\ldots+\mathbf{\Upsilon}_{q} \mathbf{d}_{t} \bigoplus_{q}+\mathbf{v}_{t},
$$

which follows from model (B.3) above with $\mathbf{b}_{j}=\mathbf{G}_{0}^{-1} \mathbf{a}_{j}, j=0,1, \mathbf{F}_{j}=\mathbf{G}_{0}^{-1} \mathbf{G}_{j}, j=1, \ldots, p, \mathbf{\Upsilon}_{j}=$ $\mathbf{G}_{0}^{-1} \boldsymbol{\Psi}_{j}, j=0,1, \ldots, q$ and $\mathbf{v}_{t}=\mathbf{G}_{0}^{-1} \mathbf{u}_{t}$, where $\mathbf{G}_{j} j=0,1, \ldots, q$, with $p$ and $q$ equal to 3 and 2 respectively. Note that the dynamic properties of model (B.6) differ from those of model (B.5), with differences in the number of cointegrating relations for certain countries required to obtain a stable solution.

\section{Appendix C: Additional Empirical Results}

\section{C.1 Conditional Forecast Probabilities}

There are discrepancies between the estimated GVAR model and the assertions implied by the forecast scenarios, mainly due to the difference in the estimation periods. To address these, we compare the conditional forecasts based on the implied GDP changes in Scenarios 0 to 2 with the unconditional

\footnotetext{
${ }^{12}$ Note that for expositional simplicity we are assuming that the PPP-GDP weights in $\widetilde{\mathbf{W}}$ are the same for all variables. In practice, as in our case, these can be somewhat different if, for example, a certain variable is excluded from the estimation of the country-specific models for certain countries (see Table B.1(ii)), as the weights then get re-weighted in order to sum to one. The same holds true for the trade weights in $\mathbf{W}_{i}$.

${ }^{13}$ All GVAR related output in this paper has been obtained using the GVAR Toolbox 2.0 of Smith and Galesi (2014) with modifications and additions to the existing functions.
} 
Scenario 0

Scenario 1

Scenario 2

$\mathrm{EU}^{+}$
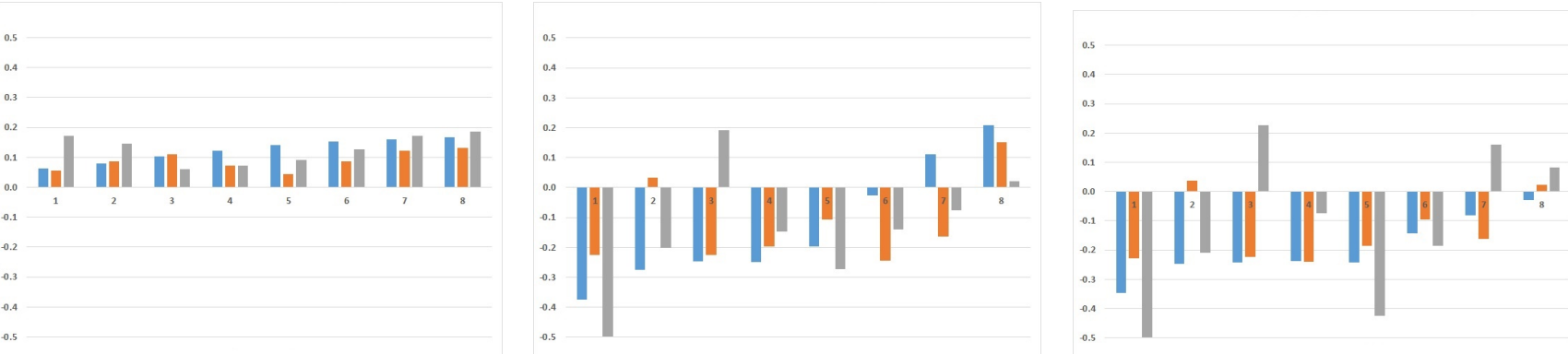

Advanced
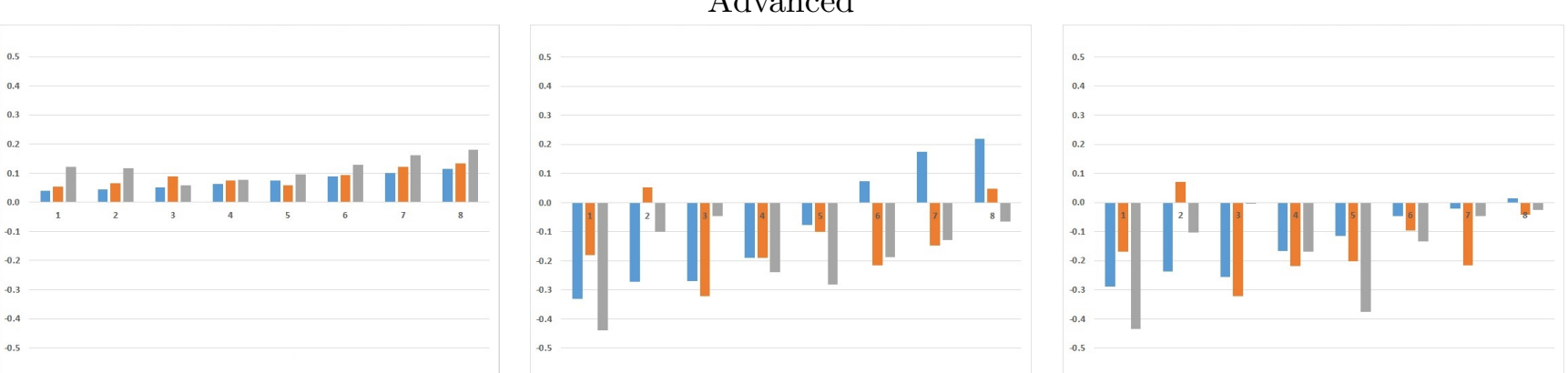

Emerging
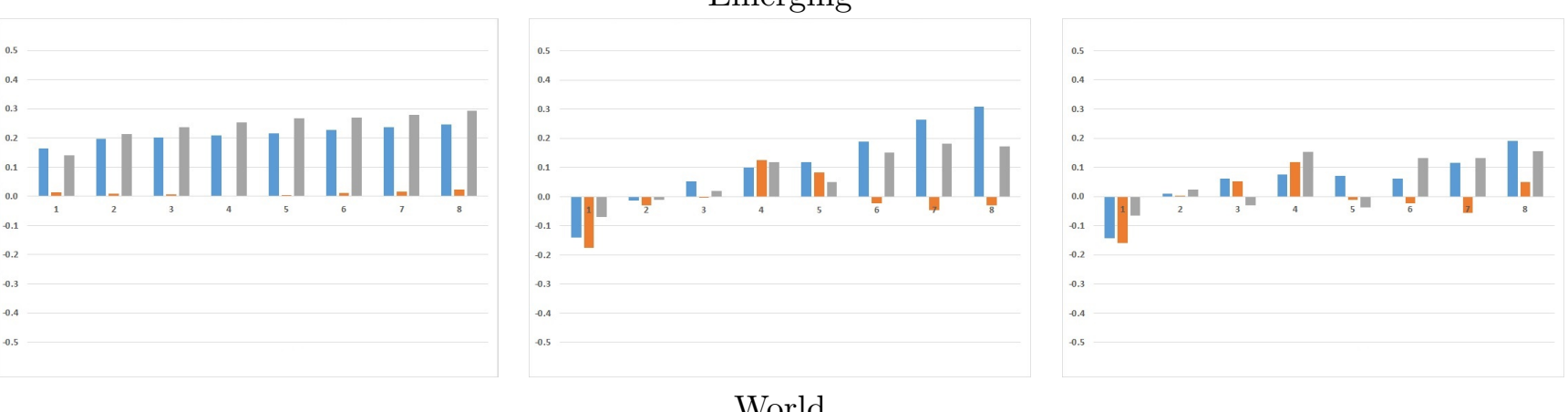

\section{World}
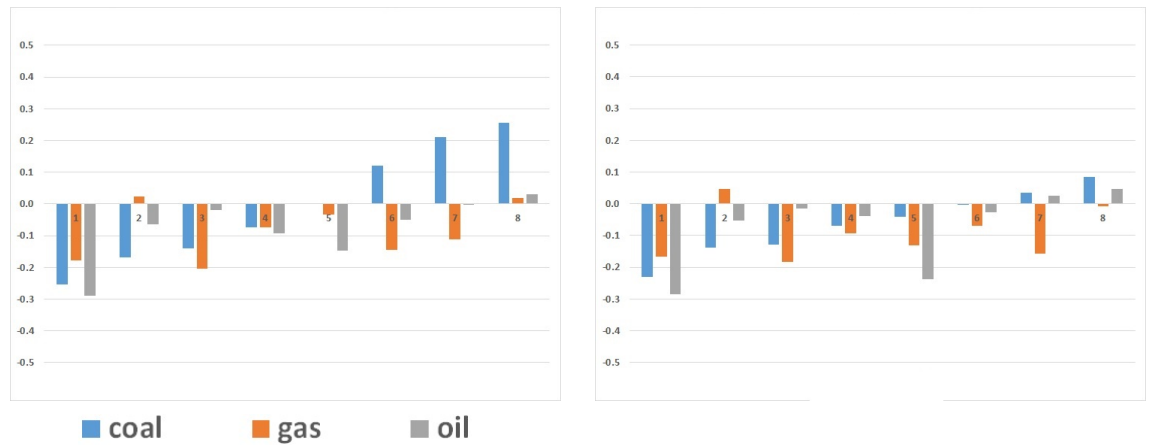

Note: The vertical axis measures the probability that consumption under the conditional forecast exceeds that under the unconditional forecast in deviation from 0.5 .

Figure C.1: Conditional forecast probabilities of changes in relative consumption by country group 
forecasts obtained from the estimated GVAR model. Figure C.1 reports the probabilities of positive consumption of the difference between the conditional and unconditional forecast of coal, natural gas, and oil by country group. The reported probabilities are the deviations from 0.5 . When the figure is positive (negative), the chance of the conditional forecast of consumption being larger (smaller) than the unconditional forecast is more than 50/50. For Scenario 0, all figures are positive and show an increasing trend over time. For the advanced and $\mathrm{EU}^{+}$countries, the figure is not very large, well below 0.2. On the other hand, the figures for the emerging economies are mostly larger than 0.2. These results suggest that, on average, the scenario growth rate is mostly higher than the estimated rate, and the discrepancy is higher for the emerging economies. With this in mind, we turn to the conditional forecast probability for Scenario 1 (against the unconditional forecast). For the $\mathrm{EU}^{+}$and advanced countries, in most quarters the chance of having negative consumption is higher. The chance of negative consumption is the highest in the first quarter with a high chance maintained over the first year, easing somewhat towards the second half of the year. For the emerging economies, after the first quarter's negative figure, chance evens out in the second and third quarters, with the figures exceeding 0.1 thereafter and rising, mainly led by coal and oil. Hence, for emerging economies the Scenario 1 effect of COVID-19 on consumption is limited to the first quarter. The result for the world is a combination of that of advanced and emerging economies. The effect of COVID-19 on the world in Scenario 1 is largely pronounced in the first quarter, with small negative effects in quarters two until four, and consumption starting to grow thereafter, in the second year. Turning to the results for Scenario 2, compared to Scenario 1 the figures for $\mathrm{EU}^{+}$and advanced countries are similarly negative during the first year but much more negative during the second year. In Scenario 2, the positive figures for the emerging economies are much smaller, especially in the second year. Consequently, the world figures are mostly negative.

\section{C.2 Forecasts of $\mathrm{CO}_{2}$ Emissions}

The conditional forecasts of the total amount of $\mathrm{CO}_{2}$ emissions for the different country groups is summarised in Figure C.2. The horizontal axis represents the quarters over the two-year forecast horizon and the vertical axis reports the forecasts of the amount of emissions. See the discussion in Section 4.2. 

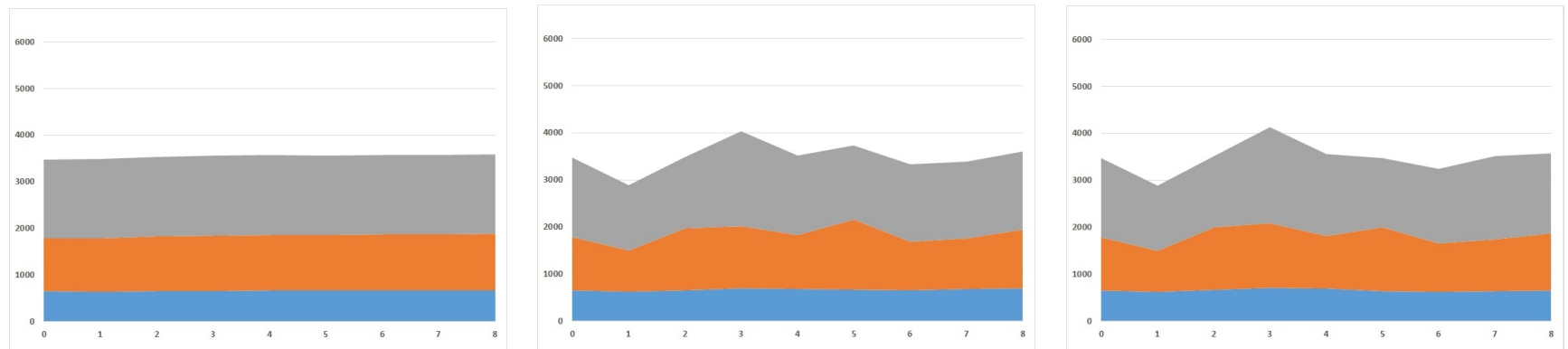

Advanced
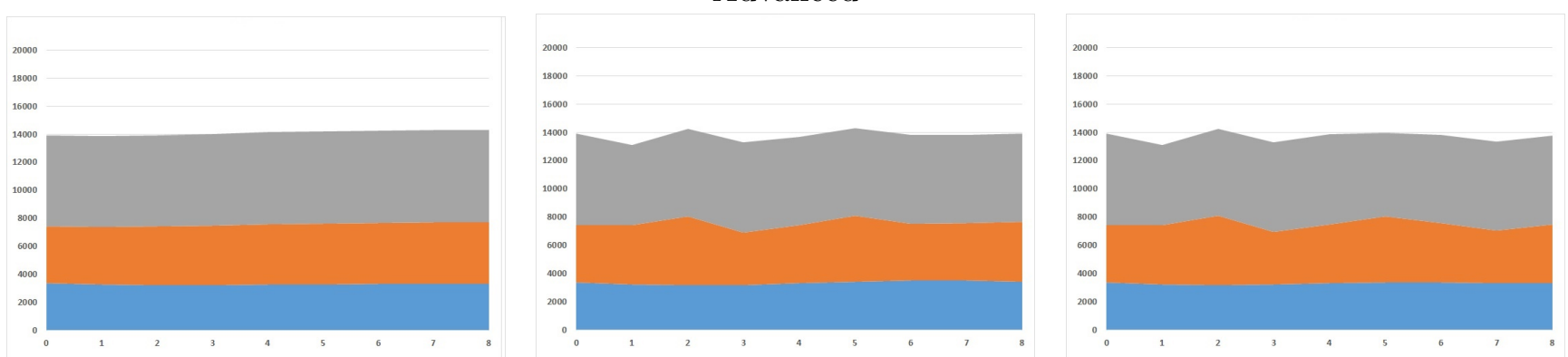

Emerging
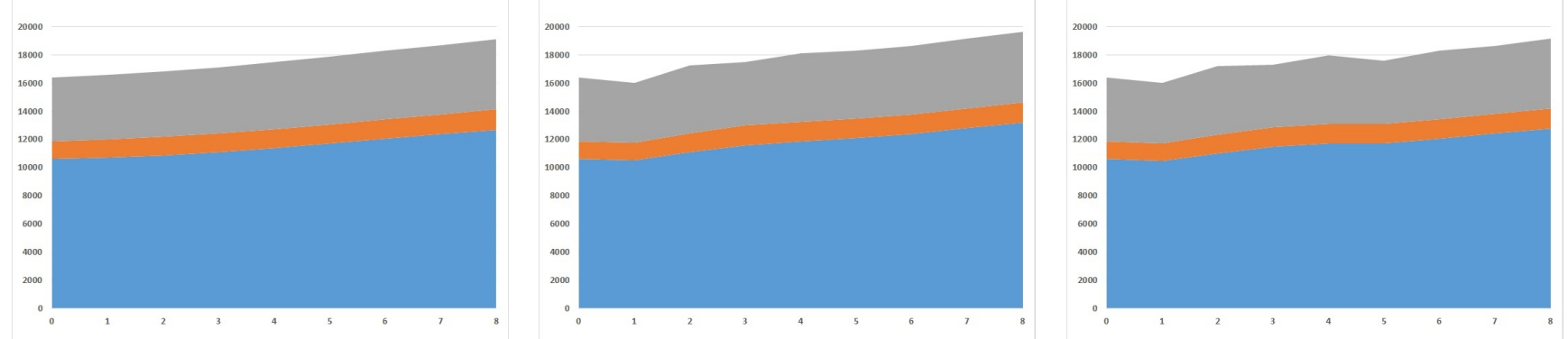

World
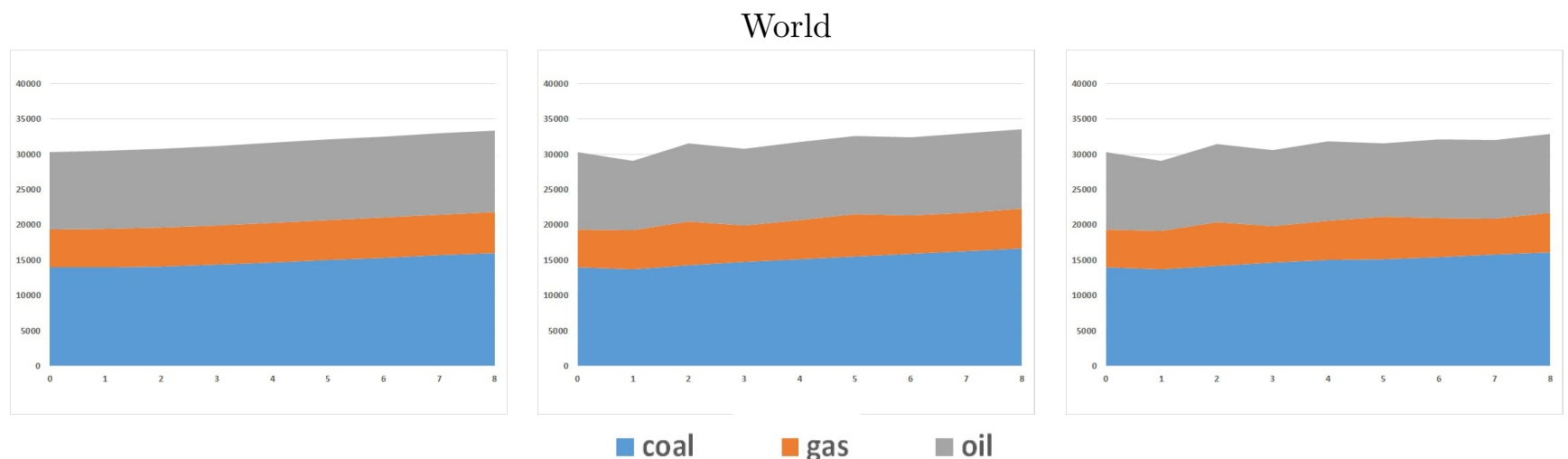

Figure C.2: Forecasts of $\mathrm{CO}_{2}$ emissions due to fossil fuel use by country group (annualised in $\mathrm{MtCO}_{2}$ )

\section{References}

Allan, J. Donovan, C. Ekins, P., Gambhir, A., Hepburn, C., Reay, D., Robins, N., Shuckburgh, E., Zenghelis, D., (2020). A net-zero emissions economic recovery from COVID-19. Working Paper No. 20-01, Oxford Smith School of Enterprise and the Environment.

Akpan, G.E., Akpan, U.F. (2012). Electricity consumption, carbon emissions and economic growth in Nigeria. International Journal of Energy Economics and Policy, 2, 292-306.

Antonakakis, N., Chatziantoniou, I., Filis, G., (2017). Energy consumption, $\mathrm{CO}_{2}$ emissions, and economic growth: An ethical dilemma. Renewable and Sustainable Energy Reviews, 68, 808-824. 
Bloch, H., Rafiq, S., Salim, R. (2012). Coal consumption, $\mathrm{CO}_{2}$ emission and economic growth in China: Empirical evidence and policy responses. Energy Economics, 34, 518-528.

Bozkurt, C., Akan, Y. (2014). Economic growth, $\mathrm{CO}_{2}$ emissions and energy consumption: The Turkish case. International Journal of Energy Economics and Policy, 4, 484-494.

BP p.l.c. (2019). BP Statistical Review of World Energy 2019, https://www.bp.com/en/global/corporate/ energy-economics/statistical-review-of-world-energy.html

Cashin, P., Mohaddes, K., Raissi, M., Raissi, M. (2014) The differential effects of oil demand and supply shocks on the global economy. Energy Economics, 44, 113-134.

Dées, S., di Mauro, F. Pesaran, M.H., Smith, L.V. (2007). Exploring the international linkages of the euro area: A global VAR analysis. Journal of Applied Econometrics, 22, 1- 38.

Elkhafif, M.A.T. (1996). An iterative approach for weather-correcting energy consumption data. Energy Economics, 18, 221-230.

Huntington, H. G., Barrios, J. J., Arora, V. (2019). Review of key international demand elasticities for major industrializing economies. Energy Policy, 133, 110878.

IMF (2020a). World Economic Outlook Update, January 2020.

IMF (2020b). World Economic Outlook, April 2020.

IMF (2020c). World Economic Outlook Update, June 2020.

Ivanov, D. (2020). Predicting the impacts of epidemic outbreaks on global supply chains: A simulationbased analysis on the coronavirus outbreak (COVID-19/SARS-CoV-2) case. Transportation Research Part E: Logistics and Transportation Review 136.

Johansen, S. (1991). Estimation and hypothesis testing of cointegration vectors in Gaussian vector autoregressive models. Econometrica, 59, 1551-1580.

Kahouli, K., (2019). Does static and dynamic relationship between economic growth and energy consumption exist in OECD countries? Energy Reports, 5, 104-116.

Le Quere, C., Jackson, R.B., Jones, M.W., Smith, A.J.P., Abernethy, S., Andrew, R.M., De-Gol1, A.J., Willis, D.R., Shan, Y., Canadell, J.G., Friedlingstein, P., Creutzig, F., Peters, G.P., (2020). Temporary reduction in daily global $\mathrm{CO}_{2}$ emissions during the COVID-19 forced confinement, Nature Climate Change, https://doi.org/10.1038/s41558-020-0797-x

Lisman, J., Sandee, J. (1964). Derivation of quarterly figures from annual data. Journal of the Royal Statistical Society (Series C), 13, 87-90.

Liu, Z., Deng, Z., Ciais, P., Lei, R., Feng, S., Davis, S.J., Wang, Y., Yue, X., Lei, Y., Zhou, H. et al., (2020). COVID-19 causes record decline in global $\mathrm{CO}_{2}$ emissions, arXiv preprint arXiv:2004.13614.

Mohaddes K., Pesaran M.H. (2016). Country-specific oil supply shocks and the global economy: a counterfactual analysis. Energy Economics, 59, 382-399.

Mohaddes K., Pesaran M.H. (2017). Oil prices and the global economy: Is it different this time around? Energy Economics, 65, 315-325.

Mohaddes K., Raissi, M. (2019). The U.S. oil supply revolution and the global economy. Empirical Economics, 57, 1515-1546.

Omri, A., Kahouli, B., (2014). Causal relationships between energy consumption, foreign direct investment and EG: Fresh evidence from dynamic simultaneous equations models. Energy Policy, 67, 913-922.

Pesaran, M. H., Schuermann, T., Weiner, S.M. (2004). Modelling regional interdependencies using a global error-correcting macroeconometric model. Journal of Business and Economics Statistics, 22, 129-162.

Pesaran, M.H., Shin, Y., Smith, R.J. (2000). Structural analysis of vector error correction models with exogenous I(1) variables. Journal of Econometrics, 97, 293-343. 
Saidi, K., Hammami, S. (2015). The impact of $\mathrm{CO}_{2}$ emissions and economic growth on energy consumption in 58 countries. Energy Reports, 1, 62-70.

Shahbaz, M., Khan, S., Tahir, M.I., (2013). The dynamic links between energy consumption, economic growth, financial development and trade in China: Fresh evidence from multivariate framework analysis. Energy Economics, 40, 8-21.

Smith, L.V., Galesi, A., (2014). The GVAR Toolbox 2.0. Available at: https://sites.google.com/site/ gvarmodelling/gvar-toolbox/

Won, S.J., Wang, X.H., Warren, H.E. (2016). Climate normals and weather normalization for utility regulation. Energy Economics, 54, 405-416. 
An Online Supplement for Assessing the Impact of COVID-19 on Global Fossil Fuel Consumption and $\mathrm{CO}_{2}$ Emissions

L. Vanessa Smith University of York

Nori Tarui

University of Hawaii

Takashi Yamagata

University of York

June 2020 


\section{S.1 Introduction}

This supplement is organised as follows: Section S.2 provides a selected set of results related to the estimated GVAR(3) model given in (8) and the associated country-specific models. Section S.3 gives the technical details associated with the GVAR forecasting discussed in Section 4.1 of the main paper.

\section{S.2 Country-Specific and GVAR results}

The estimated GVAR(3) model in $\mathbf{y}_{t}=\left(\mathbf{x}_{t}^{\prime}, \mathbf{d}_{t}^{\prime}\right)^{\prime}$, where $\mathbf{x}_{t}$ is the global variable vector and $\mathbf{d}_{t}$ is the vector of global prices, has 155 endogenous variables (3 of which correspond to the global prices), 108 stochastic trends ( 2 corresponding to the global prices) and 47 cointegrating relations ( 1 corresponding to the global prices). All the roots of the global VAR model in the 32 countries either lie on or inside the unit circle. The moduli of the largest non unit eigenvalue is 0.949 . The lag orders for the domestic variables, $p_{i}$, and foreign variables, $q_{i}$, are selected based on the Akaike criterion with $p_{\max i}=3$ and $q_{\max i}=2$. The individual country models are estimated subject to reduced rank restrictions as described in Section 3.2 and the cointegrating relations obtained are based on the trace statistic at the $95 \%$ critical value. ${ }^{14}$ For estimation, $\mathbf{x}_{i t}^{*}$ are treated as "long-run forcing" or $I(1)$ weakly exogenous with respect to the parameters of the conditional model. This assumption can be tested by regressing $\mathbf{x}_{i t}^{*}$ on the error correction terms for country $i$ and testing whether these terms are statistically significant. A selected set of results related to the estimated GVAR(3) and associated country-specific models are given in Tables S.2(i)-S.2(iv) and Figure S.2(i). Additional results are available upon request.

\footnotetext{
${ }^{14}$ The number of cointegrating relations is determined for the case where unrestricted constants and restricted trend coefficients are included in the individual country error correction models.
} 
Table S.2(i): VARX* orders and number of cointegrating relationships

\begin{tabular}{|c|c|c|c|}
\hline & VARX* & $\left.p_{i}, q_{i}\right)$ & \# Cointegrating \\
\hline Country & $p_{i}$ & $q_{i}$ & Relationships \\
\hline ARGENTINA & 3 & 1 & 1 \\
\hline AUSTRALIA & 3 & 1 & 1 \\
\hline AUSTRIA & 3 & 2 & 2 \\
\hline BELGIUM & 3 & 2 & 1 \\
\hline BRAZIL & 3 & 2 & 1 \\
\hline CANADA & 3 & 2 & 3 \\
\hline CHILE & 2 & 2 & 2 \\
\hline CHINA & 3 & 1 & 1 \\
\hline FINLAND & 3 & 1 & 1 \\
\hline FRANCE & 3 & 1 & 2 \\
\hline GERMANY & 2 & 1 & 2 \\
\hline INDIA & 3 & 2 & 2 \\
\hline INDONESIA & 3 & 1 & 3 \\
\hline ITALY & 3 & 2 & 2 \\
\hline JAPAN & 3 & 2 & 2 \\
\hline KOREA & 3 & 2 & 1 \\
\hline MALAYSIA & 3 & 1 & 3 \\
\hline HEXICO & 3 & 2 & 1 \\
\hline NETHERLANDS & 3 & 2 & 0 \\
\hline NEW ZEALAND & 1 & 1 & 2 \\
\hline NORWAY & 3 & 2 & 1 \\
\hline PHILIPPINES & 3 & 2 & 2 \\
\hline SAUDI ARABIA & 3 & 1 & 0 \\
\hline SINGAPORE & 1 & 1 & 1 \\
\hline SOUTH AFRICA & 2 & 1 & 0 \\
\hline SPAIN & 3 & 2 & 2 \\
\hline SWEDEN & 3 & 2 & 0 \\
\hline SWITZERLAND & 3 & 1 & 1 \\
\hline THAILAND & 3 & 1 & 2 \\
\hline TURKEY & 3 & 2 & 1 \\
\hline UNITED KINGDOM & 3 & 1 & 2 \\
\hline USA & 3 & 1 & 1 \\
\hline
\end{tabular}

Note: The lag orders for the domestic variables, $p_{i}$, and foreign variables, $q_{i}$, are selected based on the Akaike criterion with $p_{\max i}=3$ and $q_{\max i}=2$. The individual country models are estimated subject to reduced rank restrictions as described in Section 3.2 and the cointegrating relations obtained are based on the trace statistic at the $95 \%$ critical value. The number of cointegrating relations was reduced for certain countries based on the performance of the persistence profiles of the GVAR.

Figure S.2(i): Persistence profiles

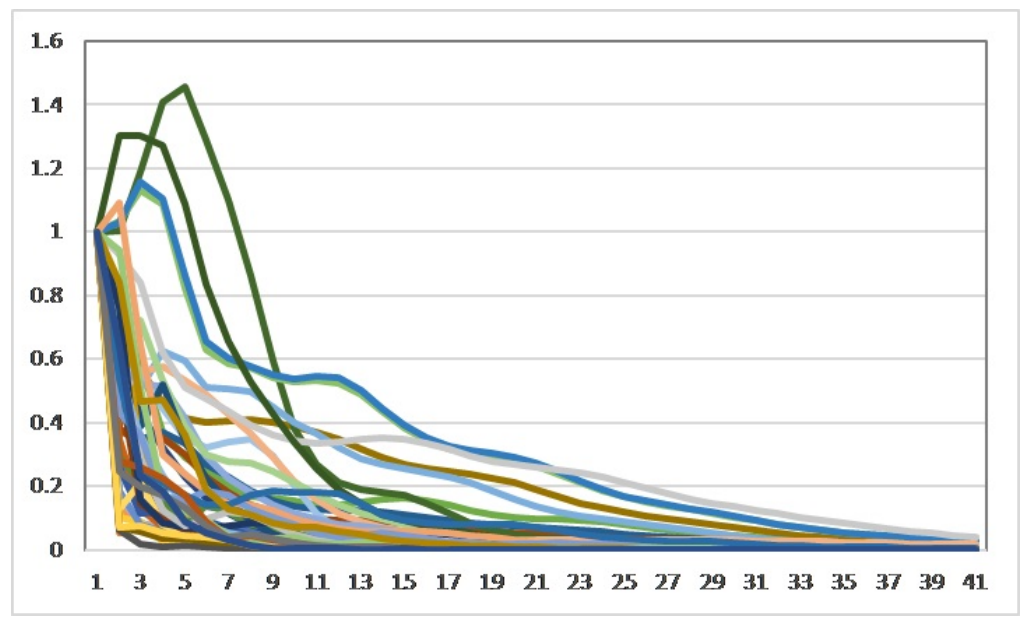


Table S.2(ii): Number of rejections of the null of parameter constancy per variable across the country specific models at the 1 percent level

\begin{tabular}{ccccccc}
\hline \hline Alternative & \multicolumn{7}{c}{ Domestic Variables } \\
Test Statistics & coal & gas & oil & $g d p$ & $e p$ & Numbers(\%) \\
\hline$P K_{\text {sup }}$ & $1(3.3)$ & $2(7.4)$ & $2(6.3)$ & $4(12.5)$ & $1(3.2)$ & $10(6.6)$ \\
$P K_{m s q}$ & $1(3.3)$ & $2(7.4)$ & $1(3.1)$ & $5(15.6)$ & $0(0.0)$ & $9(5.9)$ \\
$N$ & $4(13.3)$ & $2(7.4)$ & $3(9.4)$ & $0(0.0)$ & $0(0.0)$ & $9(5.9)$ \\
robust- $N$ & $0(0.0)$ & $1(3.7)$ & $0(0.0)$ & $0(0.0)$ & $0(0.0)$ & $1(0.7)$ \\
$Q L R$ & $7(23.3)$ & $9(33.3)$ & $7(21.9)$ & $6(18.8)$ & $2(6.5)$ & $31(20.4)$ \\
robust- $Q L R$ & $1(3.3)$ & $0(0.0)$ & $2(6.3)$ & $0(0.0)$ & $1(3.2)$ & $4(2.6)$ \\
$M W$ & $5(16.7)$ & $5(18.5)$ & $6(18.8)$ & $3(9.4)$ & $1(3.2)$ & $20(13.2)$ \\
robust- $M W$ & $1(3.3)$ & $0(0.0)$ & $2(6.3)$ & $1(3.1)$ & $0(0.0)$ & $4(2.6)$ \\
$A P W$ & $7(23.3)$ & $9(33.3)$ & $8(25.0)$ & $6(18.8)$ & $2(6.5)$ & $32(21.1)$ \\
robust- $A P W$ & $2(6.7)$ & $0(0.0)$ & $3(9.4)$ & $0(0.0)$ & $1(3.2)$ & $6(3.9)$ \\
\hline
\end{tabular}

Note: The test statistics $P K_{\text {sup }}$ and $P K_{m s q}$ are based on the cumulative sums of OLS residuals, $N$ is the Nyblom test for time-varying parameters and $Q L R, M W$ and $A P W$ are the sequential Wald statistics for a single break at an unknown change point. Statistics with the prefix robust denote the heteroskedasticity robust version of the tests. All tests are implemented at the $1 \%$ significance level.

Table S.2(iii) Contemporaneous effects of the foreign variables on their domestic counterparts in the country-specific models

\begin{tabular}{|c|c|c|c|c|}
\hline Country & coal & gas & oil & $g d p$ \\
\hline ARGENTINA & $0.31[0.23]$ & $0.08^{\dagger}[0.04]$ & $0.54[0.31]$ & $0.39[0.37]$ \\
\hline AUSTRALIA & $-0.25[0.23]$ & $-0.01[0.49]$ & $0.26[0.15]$ & $0.38^{\dagger}[0.12]$ \\
\hline AUSTRIA & $0.39[0.22]$ & $0.04[0.13]$ & $0.39^{\dagger}[0.15]$ & $0.95^{\dagger}[0.13]$ \\
\hline BELGIUM & $1.35^{\dagger}[0.34]$ & $0.00[0.16]$ & $1.08^{\dagger}[0.27]$ & $0.94^{\dagger}[0.16]$ \\
\hline BRAZIL & $0.36^{\dagger}[0.13]$ & $-0.03[0.08]$ & $-0.07[0.21]$ & $0.29[0.39]$ \\
\hline CANADA & $0.46^{\dagger}[0.14]$ & $0.37^{\dagger}[0.15]$ & $0.62^{\dagger}[0.17]$ & $0.50^{\dagger}[0.09]$ \\
\hline CHILE & $1.32^{\dagger}[0.43]$ & $-0.39[0.55]$ & $0.20[0.45]$ & $0.78^{\dagger}[0.28]$ \\
\hline CHINA & $0.04[0.04]$ & $0.01[0.04]$ & $-0.14[0.25]$ & $0.65^{\dagger}[0.31]$ \\
\hline FINLAND & $0.37[0.22]$ & $0.05[0.20]$ & $0.54^{\dagger}[0.22]$ & $1.52^{\dagger}[0.23]$ \\
\hline FRANCE & $0.01[0.12]$ & $0.60[0.31]$ & $0.51^{\dagger}[0.10]$ & $0.70^{\dagger}[0.06]$ \\
\hline GERMANY & $0.23^{\dagger}[0.06]$ & $0.27[0.17]$ & $1.09^{\dagger}[0.27]$ & $1.30^{\dagger}[0.15]$ \\
\hline INDIA & $0.00[0.04]$ & $-0.09[0.07]$ & $0.11[0.20]$ & $0.75[0.40]$ \\
\hline INDONESIA & $0.24[1.13]$ & $-0.12[0.17]$ & $1.59[0.88]$ & $0.18[0.25]$ \\
\hline ITALY & $0.82^{\dagger}[0.15]$ & $0.70^{\dagger}[0.14]$ & $0.43^{\dagger}[0.14]$ & $0.92^{\dagger}[0.13]$ \\
\hline JAPAN & $0.20^{\dagger}[0.05]$ & $0.10[0.22]$ & $0.78^{\dagger}[0.20]$ & $0.40^{\dagger}[0.19]$ \\
\hline KOREA & $0.38[0.23]$ & - & $0.46[0.26]$ & $0.27[0.24]$ \\
\hline MALAYSIA & $0.76[0.78]$ & $0.06[0.05]$ & $0.08[0.32]$ & $0.91^{\dagger}[0.14]$ \\
\hline MEXICO & $0.58^{\dagger}[0.26]$ & $0.22[0.14]$ & $0.18[0.16]$ & $0.66^{\dagger}[0.23]$ \\
\hline NETHERLANDS & $0.32^{\dagger}[0.16]$ & $0.60^{\dagger}[0.18]$ & $0.12[0.16]$ & $0.41^{\dagger}[0.11]$ \\
\hline NEW ZEALAND & $0.65[0.49]$ & $-0.16[0.34]$ & $0.39[0.34]$ & $0.36^{\dagger}[0.15]$ \\
\hline NORWAY & $0.39[0.33]$ & $0.23[0.82]$ & $0.89^{\dagger}[0.39]$ & $0.65[0.35]$ \\
\hline PHILIPPINES & $0.11[0.23]$ & - & $0.49[0.29]$ & $0.42[0.22]$ \\
\hline SAUDI ARABIA & - & $0.03[0.09]$ & $-0.28[0.18]$ & $0.37[0.24]$ \\
\hline SINGAPORE & - & $-0.93^{\dagger}[0.43]$ & $0.52^{\dagger}[0.24]$ & $1.52^{\dagger}[0.22]$ \\
\hline SOUTH AFRICA & $-0.04[0.13]$ & - & $0.18[0.27]$ & $0.34[0.10]$ \\
\hline SPAIN & $0.12[0.16]$ & $0.12[0.13]$ & $0.68^{\dagger}[0.13]$ & $0.20^{\dagger}[0.08]$ \\
\hline SWEDEN & $1.09^{\dagger}[0.34]$ & - & $1.19^{\dagger}[0.28]$ & $1.19^{\dagger}[0.17]$ \\
\hline SWITZERLAND & $0.76[0.55]$ & $0.05[0.14]$ & $0.74^{\dagger}[0.24]$ & $0.78^{\dagger}[0.17]$ \\
\hline THAILAND & $0.20[0.32]$ & $0.09[0.06]$ & $0.58^{\dagger}[0.25]$ & $0.93^{\dagger}[0.34]$ \\
\hline TURKEY & $0.23^{\dagger}[0.11]$ & - & $0.42[0.34]$ & $0.99[0.59]$ \\
\hline UNITED KINGDOM & $-0.27[0.17]$ & $0.39^{\dagger}[0.15]$ & $0.62^{\dagger}[0.14]$ & $0.42^{\dagger}[0.10]$ \\
\hline USA & $0.06[0.03]$ & $0.29[0.24]$ & $0.28^{\dagger}[0.12]$ & $0.48^{\dagger}[0.08]$ \\
\hline
\end{tabular}

Note: White's heteroskedastic-robust standard errors are given in square brackets. $\dagger$ denotes statistical significance at the $5 \%$ level. 
Table S.2(iv): F Statistics for testing the weak exogeneity of the country-specific foreign variables and global prices

\begin{tabular}{|c|c|c|c|c|c|c|c|c|c|}
\hline Country & $\mathrm{F}$ test & $\operatorname{coal}^{*}$ & gas* & oil $^{*}$ & $g d p^{*}$ & $e p^{*}$ & pcoal & pgas & poil \\
\hline ARGENTINA & $\mathrm{F}(1,96)$ & 0.01 & 1.18 & 0.12 & 1.40 & - & 0.23 & 0.14 & 1.23 \\
\hline AUSTRALIA & $\mathrm{F}(1,91)$ & 1.63 & 0.13 & 0.10 & 1.17 & - & 0.41 & 1.42 & 0.08 \\
\hline AUSTRIA & $\mathrm{F}(2,76)$ & 1.05 & 0.21 & 0.39 & 1.46 & - & 2.23 & 2.55 & 0.21 \\
\hline BELGIUM & $\mathrm{F}(1,91)$ & 0.31 & 4.61 & 0.03 & 2.00 & - & 2.43 & 2.07 & 0.44 \\
\hline BRAZIL & $\mathrm{F}(1,105)$ & 1.21 & 0.00 & 0.20 & 0.70 & - & 0.12 & 0.92 & 0.06 \\
\hline CANADA & $\mathrm{F}(3,80)$ & 0.37 & 1.17 & 1.01 & 0.08 & - & 0.67 & 2.13 & 0.39 \\
\hline CHILE & $\mathrm{F}(1,96)$ & 0.28 & 1.15 & 1.22 & 0.06 & - & 1.28 & 0.44 & 0.34 \\
\hline CHINA & $\mathrm{F}(2,90)$ & 1.75 & 0.24 & 0.77 & 0.02 & - & 0.33 & 0.00 & 0.01 \\
\hline FINLAND & $\mathrm{F}(1,96)$ & 1.74 & 0.06 & 3.25 & 2.43 & - & 0.16 & 0.01 & 1.67 \\
\hline FRANCE & $\mathrm{F}(2,90)$ & 0.09 & 0.11 & 0.29 & 0.03 & - & 0.45 & 2.25 & 0.73 \\
\hline GERMANY & $\mathrm{F}(2,90)$ & 0.47 & $3.99^{\dagger}$ & 0.11 & 0.33 & - & 1.88 & 0.97 & $4.56^{\dagger}$ \\
\hline INDIA & $\mathrm{F}(2,81)$ & 1.47 & 2.76 & 0.37 & 1.34 & - & 1.34 & 2.22 & 0.87 \\
\hline INDONESIA & $\mathrm{F}(3,94)$ & 0.61 & 0.67 & 0.26 & 0.33 & - & 0.34 & 0.23 & 0.52 \\
\hline ITALY & $\mathrm{F}(2,95)$ & 0.87 & 0.81 & 1.25 & 0.81 & - & 0.18 & 1.03 & 0.30 \\
\hline JAPAN & $\mathrm{F}(2,104)$ & 0.40 & 0.06 & 0.83 & 0.03 & - & 0.22 & 2.74 & 1.39 \\
\hline KOREA & $\mathrm{F}(1,106)$ & 0.00 & 1.22 & 0.35 & 0.51 & - & 0.85 & 0.13 & 0.00 \\
\hline MALAYSIA & $\mathrm{F}(3,94)$ & 1.18 & 1.18 & 0.95 & 0.25 & - & 0.98 & 0.40 & 0.54 \\
\hline MEXICO & $\mathrm{F}(1,96)$ & 1.19 & 0.01 & 1.15 & 1.15 & - & 0.41 & 1.26 & 2.01 \\
\hline NETHERLANDS & $\mathrm{F}(0,97)$ & - & - & - & - & - & - & - & - \\
\hline NEW ZEALAND & $\mathrm{F}(1,87)$ & 0.57 & 0.40 & 0.14 & 0.68 & - & 0.06 & 0.30 & 1.58 \\
\hline NORWAY & $\mathrm{F}(2,95)$ & 0.06 & 3.39 & 2.02 & 1.49 & - & 0.08 & 0.34 & 2.63 \\
\hline PHILIPPINES & $\mathrm{F}(2,96)$ & 1.39 & 1.56 & 0.34 & 0.49 & - & 1.23 & 0.30 & 2.33 \\
\hline SAUDI ARABIA & $\mathrm{F}(0,98)$ & - & - & - & - & - & - & - & - \\
\hline SINGAPORE & $\mathrm{F}(0,98)$ & 0.31 & 1.13 & 0.40 & 0.52 & - & 0.00 & 0.03 & 0.75 \\
\hline SOUTH AFRICA & $\mathrm{F}(1,97)$ & - & - & - & - & - & - & - & - \\
\hline SPAIN & $\mathrm{F}(2,95)$ & 1.60 & 2.48 & 0.69 & 0.00 & - & 0.32 & 0.54 & 0.07 \\
\hline SWEDEN & $\mathrm{F}(0,98)$ & - & - & - & - & - & - & - & - \\
\hline SWITZERLAND & $\mathrm{F}(1,96)$ & 0.65 & 0.76 & 0.08 & 3.09 & - & 0.01 & 0.43 & 0.41 \\
\hline THAILAND & $\mathrm{F}(2,90)$ & 0.88 & 0.16 & 0.42 & 0.05 & - & 0.30 & 0.26 & 2.35 \\
\hline TURKEY & $\mathrm{F}(1,88)$ & 1.14 & 1.82 & 1.10 & 0.10 & - & 0.22 & 0.01 & 0.11 \\
\hline UNITED KINGDOM & $\mathrm{F}(2,95)$ & 0.80 & 1.00 & 0.11 & 0.11 & - & 1.30 & 0.91 & $3.60^{\dagger}$ \\
\hline USA & $\mathrm{F}(1,80)$ & 2.31 & 0.01 & 0.09 & 0.17 & 0.28 & 2.68 & 0.00 & 1.74 \\
\hline
\end{tabular}

Note: These F statistics test zero restrictions on the coefficients of the error correction terms in the error-correction regressions for the country-specific variables. The lag orders for the domestic variables and those for the foreign and global price variables were selected based on the Akaike criterion with the maximum value for both set to two. Increasing the lag orders further reduced the number of statistically significant outcomes. ${ }^{\dagger}$ denotes statistical significance at the $5 \%$ level.

\section{S.3 Forecasting using the GVAR}

\section{S.3.1 Unconditional forecasts}

The unconditional forecasts based on the estimated GVAR(3) model in (8) are computed recursively by

$$
\boldsymbol{\mu}_{h}=\hat{\mathbf{c}}_{0}+\hat{\mathbf{c}}_{1}(T+h)+\hat{\mathbf{C}}_{1} \boldsymbol{\mu}_{h-1}+\hat{\mathbf{C}}_{2} \boldsymbol{\mu}_{h-2}+\hat{\mathbf{C}}_{3} \boldsymbol{\mu}_{h-3}, \text { for } h=1,2, \ldots, H .
$$

with initial values $\boldsymbol{\mu}_{0}=\mathbf{y}_{T}, \boldsymbol{\mu}_{-1}=\mathbf{y}_{T-1}$ and $\boldsymbol{\mu}_{-2}=\mathbf{y}_{T-2}$, where $h$ is the forecast horizon.

Equivalently expressed in terms of the GVAR(1) companion form we have

$$
\left(\begin{array}{c}
\mathbf{y}_{t} \\
\mathbf{y}_{t-1} \\
\mathbf{y}_{t-2}
\end{array}\right)=\left(\begin{array}{ccc}
\mathbf{C}_{1} & \mathbf{C}_{2} & \mathbf{C}_{3} \\
\mathbf{I}_{k+m_{d}} & \mathbf{0} & \mathbf{0} \\
\mathbf{0} & \mathbf{I}_{k+m_{d}} & \mathbf{0}
\end{array}\right)\left(\begin{array}{c}
\mathbf{y}_{t-1} \\
\mathbf{y}_{t-2} \\
\mathbf{y}_{t-3}
\end{array}\right)+\left(\begin{array}{c}
\mathbf{b}_{0}+\mathbf{b}_{1} t \\
\mathbf{0} \\
\mathbf{0}
\end{array}\right)+\left(\begin{array}{c}
\varepsilon_{t} \\
\mathbf{0} \\
\mathbf{0}
\end{array}\right)
$$

or

$$
\mathbf{Y}_{t}=\mathbf{C Y}_{t-1}+\mathbf{D}_{t}+\mathbf{E}_{t},
$$

where recall $k+m_{d}$ is the total number of domestic variables and global prices in the GVAR model.

Hence

$$
\mathbf{Y}_{T+h}=\mathbf{C}^{h} \mathbf{Y}_{T}+\sum_{\ell=0}^{h-1} \mathbf{C}^{\ell} \mathbf{D}_{T+h-\ell}+\sum_{\ell=0}^{h-1} \mathbf{C}^{\ell} \mathbf{E}_{T+h-\ell}
$$

and

$$
\mathbf{y}_{T+h}=\mathcal{F} \mathbf{Y}_{T+h},
$$


where

$$
\mathcal{F}=\left(\begin{array}{ccc}
\mathbf{I}_{k+m_{d}} & \mathbf{0}_{\left(k+m_{d}\right) \times\left(k+m_{d}\right)} & \mathbf{0}_{\left(k+m_{d}\right) \times\left(k+m_{d}\right)}
\end{array}\right) .
$$

Conditional on the initial values, $\mathbf{Y}_{T}$, the point forecasts $\boldsymbol{\mu}_{h}$ equivalently to (S.1) are given by

$$
\boldsymbol{\mu}_{h}=\mathcal{F} \mathbf{C}^{h} \mathbf{Y}_{T}+\sum_{\ell=0}^{h-1} \mathcal{F} \mathbf{C}^{\ell} \mathbf{D}_{T+h-\ell} .
$$

\section{S.3.2 Conditional forecasts}

Under joint normality of the GVAR shocks we have that $\mathbf{y}_{T+h} \mid \mathcal{I}_{T}$ is normally distributed

$$
\mathbf{y}_{T+h} \mid \mathcal{I}_{T} \sim N\left(\boldsymbol{\mu}_{h}, \boldsymbol{\Omega}_{h h}\right),
$$

where $\boldsymbol{\mu}_{h}=E\left(\mathbf{y}_{T+h} \mid \mathcal{I}_{T}\right)$ and $\boldsymbol{\Omega}_{h h}$ is given by

$$
\boldsymbol{\Omega}_{h h}=\mathcal{F} \sum_{\ell=0}^{h-1} \mathbf{C}^{\ell} \boldsymbol{\Sigma} \mathbf{C}^{\prime \ell} \mathcal{F}^{\prime},
$$

with

$$
\boldsymbol{\Sigma}=\left(\begin{array}{ccc}
\boldsymbol{\Sigma}_{\varepsilon} & \mathbf{0} & \mathbf{0} \\
\mathbf{0} & \mathbf{0} & \mathbf{0} \\
\mathbf{0} & \mathbf{0} & \mathbf{0}
\end{array}\right),
$$

and $\boldsymbol{\Sigma}_{\varepsilon}=\operatorname{Cov}\left(\boldsymbol{\varepsilon}_{T+h-i}\right)$, and $\mathbf{C}$ and $\mathcal{F}$ are given by (S.2) and (S.5), respectively. The covariance of $\boldsymbol{\varepsilon}_{t}$ can be estimated using the residuals of (8) as $\hat{\boldsymbol{\Sigma}}_{\varepsilon}=\frac{1}{T} \hat{\varepsilon}_{t} \hat{\varepsilon}_{t}^{\prime}$.

Furthermore we have

$$
\mathbf{y}_{T+h}=\boldsymbol{\mu}_{h}+\boldsymbol{\xi}_{T+h},
$$

where

$$
\boldsymbol{\xi}_{T+h}=\sum_{\ell=0}^{h-1} \mathcal{F} \mathbf{C}^{\ell} \mathbf{E}_{T+h-\ell},
$$

with $\boldsymbol{\xi}_{T+h} \mid \mathcal{I}_{T}$ normally distributed as $N\left(\mathbf{0}, \boldsymbol{\Omega}_{h h}\right)$, and $\mathbf{E}_{T+h-\ell}$ is defined by (S.2).

From (9) and (S.9) it follows that

$$
\mathbf{S} \xi_{T+j}=\mathbf{g}_{T+j}-\mathbf{S} \mu_{j} \text { for } j=1,2, \ldots, H,
$$

and setting $\mathbf{q}_{T+j}=\mathbf{g}_{T+j}-\mathbf{S} \mu_{j} \forall j$, (S.10) can be written as

$$
\left(\mathbf{I}_{H} \otimes \mathbf{S}\right) \boldsymbol{\xi}_{H}=\mathbf{q}_{H},
$$

where $\boldsymbol{\xi}_{H}=\left(\xi_{T+1}^{\prime}, \boldsymbol{\xi}_{T+2}^{\prime}, \ldots, \boldsymbol{\xi}_{T+H}^{\prime}\right)^{\prime}$ and $\mathbf{q}_{H}=\left(\mathbf{q}_{T+1}^{\prime}, \mathbf{q}_{T+2}^{\prime}, \ldots, \mathbf{q}_{T+H}^{\prime}\right)^{\prime}$.

Under joint normality of the shocks and surpressing the dependence of $\boldsymbol{\xi}_{T+h}$ on $H$ we have

$$
\begin{aligned}
& E\left(\boldsymbol{\xi}_{T+h} \mid \mathcal{I}_{T}, \mathbf{S y}_{T+j}=\mathbf{g}_{T+j}, j=1, \ldots, H\right) \\
& =E\left(\boldsymbol{\xi}_{T+h} \mid \mathcal{I}_{T},\left(\mathbf{I}_{H} \otimes \mathbf{S}\right) \boldsymbol{\xi}_{H}=\mathbf{q}_{H}\right) \\
& =\left(\mathfrak{s}_{h H}^{\prime} \otimes \mathbf{I}_{k+m_{d}}\right) \boldsymbol{\Omega}_{H}\left(\mathbf{I}_{H} \otimes \mathbf{S}^{\prime}\right)\left[\left(\mathbf{I}_{H} \otimes \mathbf{S}\right) \boldsymbol{\Omega}_{H}\left(\mathbf{I}_{H} \otimes \mathbf{S}^{\prime}\right)\right]-1 \\
& \mathbf{q}_{H},
\end{aligned}
$$

where $\mathfrak{s}_{h H}$ is a $H \times 1$ selection vector with unity as its $h^{\text {th }}$ element and zeros elsewhere, and $\boldsymbol{\Omega}_{H}$ is the $\left(k+m_{d}\right) H \times\left(k+m_{d}\right) H$ matrix

$$
\boldsymbol{\Omega}_{H}=\left(\begin{array}{cccc}
\boldsymbol{\Omega}_{11} & \boldsymbol{\Omega}_{12} & \cdots & \boldsymbol{\Omega}_{1 H} \\
\boldsymbol{\Omega}_{21} & \boldsymbol{\Omega}_{22} & \cdots & \boldsymbol{\Omega}_{2 H} \\
\vdots & \vdots & \ddots & \vdots \\
\boldsymbol{\Omega}_{H 1} & \boldsymbol{\Omega}_{H 2} & \cdots & \boldsymbol{\Omega}_{H H}
\end{array}\right)
$$

The diagonal elements of $\boldsymbol{\Omega}_{H}$, that is $\left\{\boldsymbol{\Omega}_{i i}\right\}_{i=1}^{H}$, are given by (S.7), while the off-diagonal elements can be expressed as

$$
\boldsymbol{\Omega}_{i j}= \begin{cases}\mathcal{F}\left(\sum_{\ell=0}^{i-1} \mathbf{C}^{\ell} \mathbf{\Sigma} \mathbf{C}^{\ell}\right) \mathbf{C}^{\prime(j-i)} \mathcal{F}^{\prime}, & i<j \\ \mathcal{F} \mathbf{C}^{(i-j)}\left(\sum_{\ell=0}^{j-1} \mathbf{C}^{\ell} \mathbf{\Sigma} \mathbf{C}^{\prime \ell}\right) \mathcal{F}^{\prime}, & i>j\end{cases}
$$

where $\boldsymbol{\Sigma}$ is defined by (S.8).

Hence, the conditional point forecasts are given by

$$
\boldsymbol{\mu}_{h}^{*}=\boldsymbol{\mu}_{h}+\left(\mathfrak{s}_{h H}^{\prime} \otimes \mathbf{I}_{k+m_{d}}\right) \boldsymbol{\Omega}_{H}\left(\mathbf{I}_{H} \otimes \mathbf{S}^{\prime}\right)\left[\left(\mathbf{I}_{H} \otimes \mathbf{S}\right) \boldsymbol{\Omega}_{H}\left(\mathbf{I}_{H} \otimes \mathbf{S}^{\prime}\right)\right]^{-1} \mathbf{q}_{H} .
$$




\section{S.3.3 Probability distribution of the difference between conditional and uncondi- tional forecasts}

Let $\mathbf{y}_{T+h}^{*}$ be the values of $\mathbf{y}_{T+h}$ conditional on a given GDP growth path scenario given by

$$
\mathbf{y}_{T+h}^{*}=\boldsymbol{\mu}_{h}+\boldsymbol{\xi}_{T+h}^{*}, h=1,2, \ldots, H,
$$

where $\boldsymbol{\mu}_{h}$ is given by (S.1) and $\boldsymbol{\xi}_{T+h}^{*}$ is the random variable defined by the probability distribution of $\boldsymbol{\xi}_{T+h}$ conditional on $\left(\mathbf{I}_{H} \otimes \mathbf{S}\right) \boldsymbol{\xi}_{H}=\mathbf{q}_{H}$, that is

$$
\boldsymbol{\xi}_{T+h}^{*}=\sum_{\ell=0}^{h-1} \mathcal{F} \mathbf{C}^{\ell} \mathbf{E}_{T+h-\ell} \mid\left(\mathbf{I}_{H} \otimes \mathbf{S}\right) \boldsymbol{\xi}_{H}=\mathbf{q}_{H} .
$$

The difference between $\mathbf{y}_{T+h}^{*}$ and $\mathbf{y}_{T+h}$ is given by

$$
\boldsymbol{\delta}_{T+h}=\mathbf{y}_{T+h}^{*}-\mathbf{y}_{T+h}=\boldsymbol{\xi}_{T+h}^{*}-\boldsymbol{\xi}_{T+h} .
$$

The mean difference is

$$
E\left(\boldsymbol{\delta}_{T+h} \mid \mathcal{I}_{T}\right)=\boldsymbol{\mu}_{h}^{*}-\boldsymbol{\mu}_{h} .
$$

The variance $\mathbf{V}\left(\boldsymbol{\delta}_{T+h} \mid \mathcal{I}_{T}\right)$ is given by

where $^{15}$

$$
\begin{aligned}
\mathbf{V}\left(\boldsymbol{\delta}_{T+h} \mid \mathcal{I}_{T}\right) & =\mathbf{V}\left(\boldsymbol{\xi}_{T+h}^{*} \mid \mathcal{I}_{T}\right)+\mathbf{V}\left(\boldsymbol{\xi}_{T+h} \mid \mathcal{I}_{T}\right) \\
& -\operatorname{Cov}\left(\boldsymbol{\xi}^{*}+h, \boldsymbol{\xi}_{T+h} 4 \mathcal{I}_{T}\right)-\operatorname{Cbv}\left(\boldsymbol{\xi}_{T+h}, \boldsymbol{\xi}_{T+h}^{*} \mid \mathcal{I}_{T}\right),
\end{aligned}
$$

with

$$
\mathbf{V}\left(\boldsymbol{\xi}_{T+h}^{*} \mid \mathcal{I}_{T}\right)=\mathbf{\Omega}_{h h}^{*},
$$

$$
\boldsymbol{\Omega}_{h h}^{*}=\boldsymbol{\Omega}_{h h}-\left(\mathfrak{s}_{h H}^{\prime} \otimes \mathbf{I}_{k+m_{d}}\right) \boldsymbol{\Omega}_{H}\left(\mathbf{I}_{H} \otimes \mathbf{S}^{\prime}\right)\left[\left(\mathbf{I}_{H} \otimes \mathbf{S}\right) \boldsymbol{\Omega}_{H}\left(\mathbf{I}_{H} \otimes \mathbf{S}^{\prime}\right)\right]^{-1}\left(\mathbf{I}_{H} \otimes \mathbf{S}\right) \boldsymbol{\Omega}_{H}\left(\mathfrak{s}_{h} H \otimes \mathbf{I}_{k+m_{d}}\right) .
$$

To obtain Cov $\left(\boldsymbol{\xi}_{T+h}^{*}, \boldsymbol{\xi}_{T+h} \mid \mathcal{I}_{T}\right)$, recall that conditional on $\mathcal{I}_{T}$ the $\left(k+m_{d}\right) \times 1$ random vector $\boldsymbol{\xi}_{T+h}^{*}$ defined by (S.12) is dependently distributed with mean $\boldsymbol{\mu}_{h}^{*}$ and variance $\mathbf{V}\left(\boldsymbol{\xi}_{T+h}^{*} \mid \mathcal{I}_{T}\right)=\boldsymbol{\Omega}_{h h}^{*}$. Let $\mathbf{S}_{H}^{\prime}=\boldsymbol{\Omega}_{H}^{1 / 2}\left(\mathbf{I}_{H} \otimes \mathbf{S}^{\prime}\right)$ and note that (S.15) can alternatively be expressed as

$$
\boldsymbol{\Omega}_{h h}-\boldsymbol{\Omega}_{h h}^{*}=\left(\mathfrak{s}_{h H}^{\prime} \otimes \mathbf{I}_{k+m_{d}}\right) \boldsymbol{\Omega}_{H}^{1 / 2} \mathbf{P}_{H}^{1 / 2} \boldsymbol{\Omega}_{H}^{1 / 2}\left(\mathfrak{s}_{h H} \otimes \mathbf{I}_{k+m_{d}}\right),
$$

where $\mathbf{P}_{H}=\mathbf{S}_{H}^{\prime}\left(\mathbf{S}_{H} \mathbf{S}_{H}^{\prime}\right)^{-1} \mathbf{S}_{H}$ is a projection matrix which is symmetric, idempotent and positive semi-definite. Hence, $\boldsymbol{\Omega}_{h h}-\boldsymbol{\Omega}_{h h}^{*}$ is a positive semi-definite matrix.

Under the assumption that ${ }^{16}$

$$
\operatorname{Cov}\left(\boldsymbol{\xi}_{T+h}, \boldsymbol{\xi}_{T+h}^{*} \mid \mathcal{I}_{T}\right)=\mathbf{V}\left(\boldsymbol{\xi}_{T+h}^{*} \mid \mathcal{I}_{T}\right),
$$

using this result in (S.13) the variance of $\boldsymbol{\delta}_{T+h}$ is derived as

$$
\mathbf{V}\left(\boldsymbol{\delta}_{T+h} \mid \mathcal{I}_{T}\right)=\boldsymbol{\Omega}_{h h}-\boldsymbol{\Omega}_{h h}^{*} \geq 0,
$$

which is a positive semi-definite matrix. Hence,

$$
\boldsymbol{\delta}_{T+h} \mid \mathcal{I}_{T} \sim N\left(\boldsymbol{\mu}_{h}^{*}-\boldsymbol{\mu}_{h}, \boldsymbol{\Omega}_{h h}-\boldsymbol{\Omega}_{h h}^{*}\right) .
$$

\section{References}

Pesaran, M.H., Smith, L.V., Smith, R.P. (2007). What if the UK or Sweden had joined the Euro in 1999? An empirical evaluation using a global VAR. International Journal of Finance and Economics, $12,55-87$.

\footnotetext{
${ }^{15}$ The dependence of $\boldsymbol{\Omega}_{h h}^{*}$ on $H$ is suppressed here.

${ }^{16}$ For a similar assumption and related argument see Pesaran et al. (2007).
} 


\section{S.3.3 Probability distribution of the difference between conditional and uncondi- tional forecasts}

Let $\mathbf{y}_{T+h}^{*}$ be the values of $\mathbf{y}_{T+h}$ conditional on a given GDP growth path scenario given by

$$
\mathbf{y}_{T+h}^{*}=\boldsymbol{\mu}_{h}+\boldsymbol{\xi}_{T+h}^{*}, h=1,2, \ldots, H,
$$

where $\boldsymbol{\mu}_{h}$ is given by (S.1) and $\boldsymbol{\xi}_{T+h}^{*}$ is the random variable defined by the probability distribution of $\boldsymbol{\xi}_{T+h}$ conditional on $\left(\mathbf{I}_{H} \otimes \mathbf{S}\right) \boldsymbol{\xi}_{H}=\mathbf{q}_{H}$, that is

$$
\boldsymbol{\xi}_{T+h}^{*}=\sum_{\ell=0}^{h-1} \mathcal{F} \mathbf{C}^{\ell} \mathbf{E}_{T+h-\ell} \mid\left(\mathbf{I}_{H} \otimes \mathbf{S}\right) \boldsymbol{\xi}_{H}=\mathbf{q}_{H} .
$$

The difference between $\mathbf{y}_{T+h}^{*}$ and $\mathbf{y}_{T+h}$ is given by

$$
\boldsymbol{\delta}_{T+h}=\mathbf{y}_{T+h}^{*}-\mathbf{y}_{T+h}=\boldsymbol{\xi}_{T+h}^{*}-\boldsymbol{\xi}_{T+h} .
$$

The mean difference is

$$
E\left(\boldsymbol{\delta}_{T+h} \mid \mathcal{I}_{T}\right)=\boldsymbol{\mu}_{h}^{*}-\boldsymbol{\mu}_{h} .
$$

The variance $\mathbf{V}\left(\boldsymbol{\delta}_{T+h} \mid \mathcal{I}_{T}\right)$ is given by

$$
\begin{aligned}
\mathbf{V}\left(\boldsymbol{\delta}_{T+h} \mid \mathcal{I}_{T}\right) & =\mathbf{V}\left(\boldsymbol{\xi}_{T+h}^{*} \mid \mathcal{I}_{T}\right)+\mathbf{V}\left(\boldsymbol{\xi}_{T+h} \mid \mathcal{I}_{T}\right) \\
& -\operatorname{Cov}\left(\boldsymbol{\xi}_{T+h}^{*}, \boldsymbol{\xi}_{T+h} \mid \mathcal{I}_{T}\right)-\operatorname{Cov}\left(\boldsymbol{\xi}_{T+h}, \boldsymbol{\xi}_{T+h}^{*} \mid \mathcal{I}_{T}\right)
\end{aligned}
$$

where 15

$$
\mathbf{V}\left(\boldsymbol{\xi}_{T+h}^{*} \mid \mathcal{I}_{T}\right)=\mathbf{\Omega}_{h h}^{*},
$$

with

$$
\boldsymbol{\Omega}_{h h}^{*}=\boldsymbol{\Omega}_{h h}-\left(\mathfrak{s}_{h H}^{\prime} \otimes \mathbf{I}_{k+m_{d}}\right) \boldsymbol{\Omega}_{H}\left(\mathbf{I}_{H} \otimes \mathbf{S}^{\prime}\right)\left[\left(\mathbf{I}_{H} \otimes \mathbf{S}\right) \boldsymbol{\Omega}_{H}\left(\mathbf{I}_{H} \otimes \mathbf{S}^{\prime}\right)\right]^{-1}\left(\mathbf{I}_{H} \otimes \mathbf{S}\right) \boldsymbol{\Omega}_{H}\left(\mathfrak{s}_{h H} \otimes \mathbf{I}_{k+m_{d}}\right) .
$$

To obtain $\operatorname{Cov}\left(\boldsymbol{\xi}_{T+h}^{*}, \boldsymbol{\xi}_{T+h} \mid \mathcal{I}_{T}\right)$, recall that conditional on $\mathcal{I}_{T}$ the $\left(k+m_{d}\right) \times 1$ random vector $\boldsymbol{\xi}_{T+h}^{*}$ defined by $\mathrm{S} .12$ is dependently distributed with mean $\boldsymbol{\mu}_{h}^{*}$ and variance $\mathbf{V}\left(\boldsymbol{\xi}_{T+h}^{*} \mid \mathcal{I}_{T}\right)=\boldsymbol{\Omega}_{h h}^{*}$. Let $\mathbf{S}_{H}^{\prime}=\boldsymbol{\Omega}_{H}^{1 / 2}\left(\mathbf{I}_{H} \otimes \mathbf{S}^{\prime}\right)$ and note that $\mathrm{S.15}$ ) can alternatively be expressed as

$$
\boldsymbol{\Omega}_{h h}-\boldsymbol{\Omega}_{h h}^{*}=\left(\mathfrak{s}_{h H}^{\prime} \otimes \mathbf{I}_{k+m_{d}}\right) \boldsymbol{\Omega}_{H}^{1 / 2} \mathbf{P}_{H}^{1 / 2} \boldsymbol{\Omega}_{H}^{1 / 2}\left(\mathfrak{s}_{h H} \otimes \mathbf{I}_{k+m_{d}}\right),
$$

where $\mathbf{P}_{H}=\mathbf{S}_{H}^{\prime}\left(\mathbf{S}_{H} \mathbf{S}_{H}^{\prime}\right)^{-1} \mathbf{S}_{H}$ is a projection matrix which is symmetric, idempotent and positive semi-definite. Hence, $\boldsymbol{\Omega}_{h h}-\boldsymbol{\Omega}_{h h}^{*}$ is a positive semi-definite matrix.

Under the assumption that 16

$$
\operatorname{Cov}\left(\boldsymbol{\xi}_{T+h}, \boldsymbol{\xi}_{T+h}^{*} \mid \mathcal{I}_{T}\right)=\mathbf{V}\left(\boldsymbol{\xi}_{T+h}^{*} \mid \mathcal{I}_{T}\right),
$$

using this result in $\mathrm{S} .13$ the variance of $\boldsymbol{\delta}_{T+h}$ is derived as

$$
\mathbf{V}\left(\boldsymbol{\delta}_{T+h} \mid \mathcal{I}_{T}\right)=\boldsymbol{\Omega}_{h h}-\boldsymbol{\Omega}_{h h}^{*} \geq 0,
$$

which is a positive semi-definite matrix. Hence,

$$
\boldsymbol{\delta}_{T+h} \mid \mathcal{I}_{T} \sim N\left(\boldsymbol{\mu}_{h}^{*}-\boldsymbol{\mu}_{h}, \boldsymbol{\Omega}_{h h}-\boldsymbol{\Omega}_{h h}^{*}\right) .
$$

\section{References}

Pesaran, M.H., Smith, L.V., Smith, R.P. (2007). What if the UK or Sweden had joined the Euro in 1999? An empirical evaluation using a global VAR. International Journal of Finance and Economics, $12,55-87$.

\footnotetext{
${ }^{15}$ The dependence of $\boldsymbol{\Omega}_{h h}^{*}$ on $H$ is suppressed here.

${ }^{16}$ For a similar assumption and related argument see Pesaran et al. (2007).
} 